\title{
MEDIAN SPACES AND SPACES WITH MEASURED WALLS
}

\author{
INDIRA CHATTERJI, CORNELIA DRUŢU, AND FRÉDÉRIC HAGLUND
}

\begin{abstract}
We prove the existence of a close connection between spaces with measured walls and median metric spaces. In particular we show that some spaces with measured walls (e.g. real hyperbolic spaces) embed into median metric spaces which are at finite Hausdorff distance from their embedded image.

We also relate properties ( $\mathrm{T}$ ) and Haagerup (a-T-menability) to actions on median spaces and on spaces with measured walls. This allows us to explore the relationship between the classical properties $(\mathrm{T})$ and Haagerup and their versions concerning affine isometric actions on $L^{p}$-spaces.
\end{abstract}

\section{Contents}

1. Introduction. 2

2. Median spaces and their geometry. 6

2.1. Definitions and examples. 6

2.2. Convexity and gate property in median spaces. 10

2.3. Approximate geodesics and medians; completions of median spaces $\quad 12$

2.4. Rectangles and parallel pairs. 13

3. The median space associated to a space with measured walls. 16

3.1. Preliminaries on measures. 16

3.2. Spaces with measured walls. 17

3.3. Embedding a space with measured walls in a median space. 20

3.4. Distances to the associated median space. 22

4. A review of median algebras. 25

4.1. Definitions, examples. 25

4.2. Convexity 26

5. From median spaces to spaces with measured walls. 27

6. Kernels, median spaces, property (T) and a-T-menability 32

6.1. Different types of kernels 32

6.2. Left invariant kernels, property (T) and a-T-menability 35

7. Quasifications and further examples. 39

Date: July 6, 2007.

2000 Mathematics Subject Classification. Primary 20F65; Secondary 46B04, 20F67, 22F50.

Key words and phrases. spaces with measured walls, median metric spaces, Haagerup property, prop$\operatorname{erty}(\mathrm{T})$. 


\section{INTRODUCTION.}

We study the geometric notion of median space, a metric space for which, given any triple of points, there exists a unique median point, that is a point which is simultaneously between any two points in that triple. A point $x$ is said to be between two other points $a, b$ in a metric space $(X, \operatorname{dist})$ if $\operatorname{dist}(a, x)+\operatorname{dist}(x, b)=\operatorname{dist}(a, b)$.

Examples of median spaces are trees, sets of vertices of simplicial trees, $\mathbb{R}^{n}$ with the $\ell^{1}$ metric for any $n \geq 1, \operatorname{CAT}(0)$ cube complexes on which we replace the Euclidean metric on cubes by the $\ell^{1}$ metric, 0 -skeleta of CAT(0) cube complexes as above (with the induced metric). According to Chepoi [Che00] the class of 1-skeleta of CAT(0) cube complexes coincides with the class of median graphs (simplicial graphs whose combinatorial distance is median). See also [Ger97] and [Ger98] for a similar equivalence. Thus, median spaces can be thought of as generalizations to non-discrete cases of 0 -skeleta of $\operatorname{CAT}(0)$ cube complexes, in the same spirit in which real trees have generalized the notion of simplicial tree.

The ternary algebra naturally associated with a median space is called a median algebra. These were studied in [dV93], [BH83], [Isb80], [Sho54a], [Sho54b]. More geometrical studies of median spaces were started in [Rol98] or [Nic]. In this article, we emphasize that a median space has a richer structure than the algebraic one, and we use this structure to study groups.

Nica in [Nic04], and Niblo and the first author in [CN04] proved simultaneously and independently an equivalence of category between CAT(0) cube complexes, possibly infinite dimensional, and discrete spaces with walls (notion introduced by F. Paulin and the third author in [HP98]). Discrete spaces with walls were generalized by Cherix, Martin and Valette in [CMV04] to spaces with measured walls. It turns out that in some sense the category of spaces with measured walls is equivalent to the category of general median spaces. More precisely, we prove the following:

Theorem 1.1. (1) Any space with measured walls embeds isometrically in a "naturally" associated median space. Moreover, any homomorphism between two spaces with measured walls induces an isometry between the associated median spaces.

(2) Conversely, any median space ( $X$, dist) is a space with measured walls and the wall metric coincides with the original metric. Moreover any isometry between median spaces induces an isomorphism between the structures of measured walls.

(3) In particular, for any median space $(X$, dist) there exists a measured space $(\mathcal{W}, \mu)$ such that $\left(X\right.$, dist) embeds isometrically in $L^{1}(\mathcal{W}, \mu)$.

The naturality of the medianization of a space with measured walls defined by Theorem 1.1 needs deeper explanations than what we are able to give in this introduction. 
Nevertheless let us mention that, under some extra assumptions, a space with measured walls is at finite Hausdorff distance of its associated median space, a result inspired by Ch. Hruska and D. Wise's work [HW05].

Theorem 1.2 (Theorem 3.24). Let $(X, \mathcal{W}, \mathcal{B}, \mu)$ be a space with measured walls, that is $\mu$-locally finite in the sense of Definition 3.21, and such that the wall pseudo-distance $\operatorname{pdist}_{\mu}(x, y)=\mu(\mathcal{W}(x \mid y))$ is $Y_{\delta}$ in the sense of Definition 3.23.

Then the Hausdorff distance from the space $X$ to the associated median space is finite.

A consequence of Theorem 1.2 is the following.

Corollary 1.3. The real hyperbolic space $\mathbb{H}^{n}$ embeds isometrically into a median space which is at finite Hausdorff distance from the embedded $\mathbb{H}^{n}$.

In particular, the full isometry group of the real hyperbolic space $\mathbb{H}^{n}$ acts properly and cocompactly on the (locally compact) median space associated with the usual measured walls structure on $\mathbb{H}^{n}$. Thus the class of groups acting geometrically on proper median spaces includes all the uniform lattices of real hyperbolic spaces. According to Sageev [Sag95], if the fundamental group of a closed hyperbolic 3-manifold $M$ acts effectively and by isometries on a finite dimensional CAT(0) cube complex, then $M$ contains an immersed incompressible surface. In view of Sageev's and Chepoi's Theorems, Corollary 1.3 can be thought as an evidence for the virtual Hacken conjecture. But in order to actually prove the conjecture, one should either be able to extract a median $G$-graph out of a median $G$-space, or generalize Sageev's techniques to median spaces.

Theorem 1.1 allows us to prove the following median characterizations of Kazhdan's property (T) and Haagerup's property (also called a-T-menability):

Theorem 1.4. Let $G$ be a locally compact second countable group.

(1) The group $G$ has property $(T)$ if and only if any action by isometries on a median space has bounded orbits.

(2) The group $G$ is a-T-menable if and only if it admits a proper action by isometries on a median space.

Previously, Niblo and Reeves proved that if a discrete countable group has a proper action on a finite dimensional CAT(0) cubical complex then the group is a-T-menable, and that if a group has property $(\mathrm{T})$ then any action on a finite dimensional $\mathrm{CAT}(0)$ cubical complex has a global fixed point ([NR97], [NR98]). Later Nica proved [Nic] that countable groups with property $(\mathrm{T})$ cannot act on median spaces with an unbounded orbit.

Also in relation with Theorem 1.4, Guentner and Higson showed that a countable discrete group acting properly on a finite dimensional CAT(0) cubical complex is weakly amenable with Cowling-Haagerup constant 1. On the other hand, M. Cowling conjectured that a countable discrete group is a-T-menable if and only if it is weakly amenable with Cowling-Haagerup constant $1\left[\mathrm{CCJ}^{+} 01, \S 1.3 .1\right]$. Theorem 1.4, (2), is related to 
one implication in Cowling's conjecture. Indeed, if for a discrete countable a-T-menable group it would be possible (eventually under some extra-hypotheses) to extract from its proper action on a median space a proper action on a median graph which is the 1skeleton of a finite dimensional CAT(0) cubical complex, then by Guentner and Higson weak amenability would follow.

Theorem 1.4 can be reformulated in terms of spaces with measured walls as follows:

Theorem 1.5. Let $G$ be a locally compact second countable group.

(1) The group $G$ has property ( $T$ ) if and only if any action by automorphisms on a space with measured walls has a bounded orbit.

(2) The group $G$ is a-T-menable if and only if it admits a proper action by automorphisms on a space with measured walls.

The equivalence in Theorem 1.5, (2), improves the result of Cherix, Martin and Valette [CMV04], who showed the same equivalence for discrete groups.

Using Theorem 1.5 and a construction from [CMV04] and [dCTV06] associating to every action on a space with measured walls an affine isometric action on an $L^{p}$-space, the following can be obtained.

Corollary 1.6. Let $G$ be a second countable locally compact group.

(1) If there exists $p>0$ such that any action of $G$ on a space $L^{p}(X, \mu)$ has bounded orbits (equivalently, for $p>1$, a fixed point) then $G$ has property $(T)$.

(2) If $G$ is a-T-menable then for every $p>0$ the group $G$ has a proper action on a space $L^{p}(X, \mu)$.

De Cornulier, Tessera and Valette proved the implication in (2) for countable discrete groups [dCTV06, Proposition 3.1].

The following converse statements hold as a consequence of well-known results relating properties $(\mathrm{T})$ and Haagerup to left-invariant conditionally negative definite kernels.

Corollary 1.7. (Corollary 6.20) Let $G$ be a second countable, locally compact group.

(1) If the group $G$ has property $(T)$ then for every $p \in(0,2]$, every continuous action by isometries of $G$ on a subset of a space $L^{p}(X, \mu)$ has bounded orbits.

(2) If for some $p \in(0,2]$, there exists a continuous proper action by isometries of $G$ on a subset of some space $L^{p}(X, \mu)$ then the group $G$ has the Haagerup property.

In [Now06] the following result has been announced: a second countable locally compact group is a-T-menable if and only if for some (for all) $p \in(1,2)$ the group has a proper affine isometric action on $L^{p}[0,1]$. The converse implication is a consequence of well-known results, hence the proof of the previous equivalence reduces to the proof of the direct implication. This proof contains an error in [Now06], coming from the fact that while $\ell_{2}$ can be seen as a subspace in $L^{p}[0,1]$ having a complementary $V$, there is a priori no way of extending an isometric action on $\ell_{2}$ to an isometric action on $L^{p}[0,1]$ (because there is no way to relate the restriction of the norm on $\ell^{2}$ and on $V$ to the norm on the whole of $\left.L^{p}[0,1]\right)$. 
Remark 1.8. In [BFGM07, §3.c], by an argument attributed to D. Fisher and G. Margulis, it is proved that for any group $G$ with property $(T)$ there exists $\varepsilon=\varepsilon(G)$ such that for every $p \in[1,2+\varepsilon)$, every action by isometries of $G$ on every space $L^{p}(X, \mu)$ has bounded orbits (i.e. a fixed point for every $p \in(1,2+\varepsilon)$ ).

Remark 1.9. For $p \gg 2$ the statements in Corollary 1.6 cannot become equivalences. Indeed, it follows from results of P. Pansu [Pan95] that the group $G=S p(n, 1)$ has fixed-point-free actions by affine isometries on $L^{p}(G)$ for $p>4 n+2$.

More recently, Y. de Cornulier, R. Tessera and A. Valette proved in [dCTV06] that any simple algebraic group of rank one over a local field has a proper affine action on a space $L^{p}(G)$ for $p$ large enough. In particular, $G=S p(n, 1)$ acts properly isometrically on $L^{p}(G)$ for $p>4 n+2$.

Also, results of M. Bourdon and H. Pajot [BP03] imply that non-elementary hyperbolic groups have fixed-point-free isometric actions on spaces $\ell^{p}$ for $p$ large enough.

G. Yu later proved [Yu05] that every discrete hyperbolic group $G$ admits a proper isometric action on the space $\ell^{p}(G \times G)$ for $p$ large enough. In particular this holds for hyperbolic groups with property $(\mathrm{T})$.

The above quoted results of Y. de Cornulier, R. Tessera and A. Valette, and of G. Yu, illustrate that neither of the two converse implications in Corollary 1.6 hold for $p \gg 2$. This shows that for every $p>2$ the fixed point property for affine isometric actions on $L^{p}$-spaces defines an a priori stronger version of property $(\mathrm{T})$, which may be called property $(\mathrm{T})_{p}$, for instance. Also, the property of having a proper affine isometric action on an $L^{p}$-space defines a weaker version of a-T-menability, which may similarly be called a- $\mathrm{T}_{p}$-menability.

Question 1.10. Can Corollary 1.6 be generalized to: "for every $p \geq q \geq 2$ property $(\mathrm{T})_{p}$ implies property $(\mathrm{T})_{q} "$ ?

Are different properties $(\mathrm{T})_{p}$ and $(\mathrm{T})_{q}$ with $p, q \gg 2$ equivalent?

What is the relation between $(\mathrm{T})_{p}$ with $p \gg 2$ and other strong versions of property (T) defined in terms of uniformly convex Banach spaces, like for instance the one defined in [Laf] ?

Note that on one hand lattices in higher rank semisimple groups have property $(\mathrm{T})_{p}$ for all $p \geq 1$, by results of Bader, Furman, Gelander and Monod [BFGM07]. On the other hand, V. Lafforgue proves that for every local field $F$ and every $F$-algebraic almost simple group $G$ whose Lie algebra contains $s l_{3}(F), G(F)$ and all its cocompact lattices have his strong version of property $(\mathrm{T})$.

Note also that the other possible version of property $(\mathrm{T})$ for $L^{p}$-spaces, starting from the "almost invariant vectors imply invariant vectors" definition of property $(\mathrm{T})$, behaves quite differently with respect to the standard property (T) [BFGM07]. This shows in particular that the two definitions are no longer equivalent in the setting of $L^{p}$ spaces.

We prove Theorems 1.4 and 1.5 using median definite kernels, that are generalizations of Robertson-Steger measure definite kernels [RS98]. Along the way this allows us to: 
- answer Robertson-Steger question [RS98, Question (i)] whether measure definite kernels can be given an intrinsic characterization among the conditionally negative definite kernels (Proposition 6.14);

- generalize to locally compact second countable groups Robertson-Steger's dynamical characterization of property (T) [RS98] and give the a-T-menability a dynamical characterization as well (Theorem 6.26).

In the last section we describe some generalizations of median spaces, further examples, and we study the behavior of the median structure and of the structure of measured walls with respect to ultralimits and asymptotic cones.

Acknowledgments: Part of the work on the present paper was carried out during visits to the Universities of Paris XI (Paris-Sud) and Lille 1. The authors would like to express their gratitude towards these institutions.

The first author is thankful to the FIM in Zürich for its hospitality, during part of the work on this paper. The second author thanks the Centre Interfacultaire Bernoulli in Lausanne for its hospitality during the final stages of the work on the paper. The third author wishes to thank his colleague Alano Ancona for numerous discussions on measures on $\sigma$-algebras generated by semi-rings.

\section{Median SPACES AND THeir GeOMetry.}

\subsection{Definitions and examples.}

Definition 2.1 (pseudo-metric spaces). A pseudo-metric or pseudo-distance on a set $X$ is a symmetric map pdist : $X \times X \rightarrow \mathbb{R}_{+}$satisfying the triangle inequality. Distinct points $x \neq y$ with $\operatorname{pdist}(x, y)=0$ are allowed.

A map $f:\left(X_{1}\right.$, pdist $\left._{1}\right) \rightarrow\left(X_{2}\right.$, pdist $\left._{2}\right)$ is an isometry if $\operatorname{pdist}_{2}(f(x), f(y))=\operatorname{pdist}_{1}(x, y)$ (note that $f$ is not necessarily injective).

A space $X$ with a pseudo-metric pdist has a canonical metric quotient $\widehat{X}=X / \sim$ composed of the equivalence classes for the equivalence relation $x \sim y \Leftrightarrow \operatorname{pdist}(x, y)=0$, endowed with the metric $\operatorname{dist}(\hat{x}, \hat{y})=\operatorname{pdist}(x, y)$. We call $\widehat{X}$ the metric quotient of $X$. The natural map $X \rightarrow \widehat{X}$ is an isometry.

If $x$ is a point in $X$ and $r \geq 0$ then $B(x, r)$ denotes the open ball of radius $r$ around $x$ in $X$, that is the set $\{y \in X ; \operatorname{pdist}(y, x)<r\} ; \bar{B}(x, r)$ denotes the closed ball of radius $r$ around $x$, that is the set $\{y \in X ; \operatorname{pdist}(y, x) \leq r\}$.

For every $Y \subseteq X$ and $r \geq 0$, we denote by $\overline{\mathcal{N}}_{r}(Y)$ the open $r$-tubular neighborhood of $Y$ in $X$, that is $\{y \in X ; \operatorname{pdist}(y, Y)<r\}$; we denote by $\overline{\mathcal{N}}_{r}(Y)$ the closed $r$-tubular neighborhood of $Y$ in $X,\{y \in X ; \operatorname{pdist}(y, Y) \leq r\}$.

Definition 2.2 (intervals). Let ( $X$, pdist) be a pseudo-metric space. A point $b$ is between $a$ and $c$ if pdist $(a, b)+\operatorname{pdist}(b, c)=\operatorname{pdist}(a, c)$. We denote by $I(a, c)$ the set of points that are between $a$ and $c$, and we call $I(a, c)$ the interval between $a$ and $c$. A finite sequence 
of points $\left(a_{1}, a_{2}, \ldots, a_{n}\right)$ is called geodesic sequence if and only if

$$
\operatorname{pdist}\left(a_{1}, a_{n}\right)=\operatorname{pdist}\left(a_{1}, a_{2}\right)+\operatorname{pdist}\left(a_{2}, a_{3}\right)+\cdots+\operatorname{pdist}\left(a_{n-1}, a_{n}\right) .
$$

So $(a, b, c)$ is a geodesic sequence if and only if $b \in I(a, c)$.

Definition 2.3 (median point). Let $a, b, c$ be three points of a pseudo-metric space (X, dist). We denote the intersection $I(a, b) \cap I(b, c) \cap I(a, c)$ by $M(a, b, c)$, and we call any point in $M(a, b, c)$ a median point for $a, b, c$. We note that $I(a, b)=\{x \in X, x \in$ $M(a, x, b)\}$.

Definition 2.4 (median spaces). A median (pseudo-)metric space is a (pseudo-)metric space in which for any three points $x, y, z$ the set $M(x, y, z)$ is non-empty and of diameter zero (any two median points are at pseudo-distance 0). In particular a metric space is median if any three points $x, y, z$ have one and only one median point, which we will denote by $m(x, y, z)$.

Note that a pseudo-metric space is median if and only if its metric quotient is median.

A strict median subspace of a median pseudo-metric space $(X$, dist) is a subset $Y$ of $X$ such that for any three points $x, y, z$ in $Y$, the set $M(x, y, z)$ is contained in $Y$.

A subset $Y \subset X$ is a median subspace if for any three points $x, y, z$ in $Y$, we have $M(x, y, z) \cap Y \neq \emptyset$. Note that $Y$ is then median for the induced pseudo-metric. An intersection of strict median subspaces is obviously a strict median subspace, thus we may define the strict median hull of any subset $Y \subset X$. When $X$ is a metric space median subspaces are strict, thus we simplify the terminology to median hull.

A homomorphism of median pseudo-metric spaces is a map $f: X_{1} \rightarrow X_{2}$ between two median pseudo-metric spaces such that for any three points $x, y, z \in X_{1}$ we have $f\left(M_{X_{1}}(x, y, z)\right) \subset M_{X_{2}}(f(x), f(y), f(z))$. This is equivalent to asking that $f$ preserves the betweenness relation, that is $f(I(a, b)) \subset I(f(a), f(b))$.

Remark 2.5. A median metric space together with the ternary operation $(x, y, z) \mapsto$ $m(x, y, z)$ is a particular instance of what is called a median algebra (see Example 4.5 in Section 4). We will use freely some classical results in the theory of abstract median algebras - although it is not difficult to prove them directly in our geometric context.

Convention 2.6. Throughout the paper, we will call median metric spaces simply median spaces.

Definition 2.7. We say that a metric space ( $X$, dist) is submedian if it admits an isometric embedding into a median space.

Here are the main examples we have in mind.

Examples 2.8. (1) On the real line $\mathbb{R}$, the median function is just taking the middle point of a triple, that is $m_{\mathbb{R}}(a, b, c)=a+b+c-[\max (a, b, c)+\min (a, b, c)]$. More generally, $\mathbb{R}^{n}$ with the $\ell_{1}$ norm is a median space and

$$
m(\vec{x}, \vec{y}, \vec{z})=\left(m_{\mathbb{R}}\left(x_{1}, y_{1}, z_{1}\right), \ldots, m_{\mathbb{R}}\left(x_{n}, y_{n}, z_{n}\right)\right) .
$$


The interval between two points $\vec{x}, \vec{y} \in \mathbb{R}^{n}$ is the $n$-cube with opposite corners $\vec{x}$ and $\vec{y}$ and edges parallel to the coordinate axes.

(2) The $\ell_{1}$-product of two pseudo-metric spaces $\left(X_{1}\right.$, pdist $\left._{1}\right)$ and $\left(X_{2}\right.$, pdist $\left._{2}\right)$ is the set $X_{1} \times X_{2}$, endowed with the pseudo-metric

$$
\operatorname{pdist}\left(\left(x_{1}, x_{2}\right),\left(y_{1}, y_{2}\right)\right)=\operatorname{pdist}_{1}\left(x_{1}, y_{1}\right)+\operatorname{pdist}_{2}\left(x_{2}, y_{2}\right) \text {. }
$$

Then $\left(X_{1} \times X_{2}\right.$,pdist $)$ is median if and only if $\left(X_{1}\right.$, pdist 1$)$ and $\left(X_{2}\right.$, pdist $\left._{2}\right)$ are median (the components of a median point in $X_{1} \times X_{2}$ are median points of the components).

(3) (trees) Every $\mathbb{R}$-tree is a median space.

(4) (motivating example: CAT(0) cube complexes) The 1-skeleton of a CAT(0) cube complex is a (discrete) median space. In fact, according to [Che00, Theorem 6.1] a simplicial graph is median if and only if it is the 1-skeleton of a CAT(0) cube complex.

(5) A discrete space with walls (in the sense of [HP98]) is submedian by [CN04] and [Nic04]. We shall prove further in this paper that actually a space is submedian if and only if it is a space with measured walls (see Corollary 5.4).

Various examples of submedian spaces can also be deduced from Remark 6.9.

(6) ( $L^{1}$-spaces) Given a measured space $(X, \mathcal{B}, \mu)$, the metric space $L^{1}(X, \mu)$ is median. Indeed, it is enough to see that the real vector space $\mathcal{L}^{1}(X, \mu)$ of measurable functions $f: X \rightarrow \mathbb{R}$ with finite $L^{1}$-norm is a median pseudo-metric space. Define on $\mathcal{L}^{1}(X, \mu)$ a ternary operation $(f, g, h) \mapsto m(f, g, h)$ by

$$
m(f, g, h)(x)=m_{\mathbb{R}}(f(x), g(x), h(x)) .
$$

Clearly $m=m(f, g, h)$ is measurable and since it is pointwise between $f$ and $g$, it satisfies $\|f-g\|_{1}=\|f-m\|_{1}+\|m-g\|_{1}$. In particular $m \in \mathcal{L}^{1}(X, \mu)$ and $m \in I(f, g)$, where the interval is defined with respect to the pseudo-distance $\operatorname{pdist}(f, g)=\|f-g\|_{1}$. Similarly we have $m \in I(g, h)$ and $m \in I(f, h)$, so that $m(f, g, h)$ is a median point for $f, g, h$.

It is easy to see that a function $p \in \mathcal{L}^{1}(X, \mu)$ belongs to $I(f, g)$ if and only if the set of points $x$ such that $p(x)$ is not between $f(x)$ and $g(x)$ has measure 0 . It follows that $M(f, g, h)$ is the set of functions that are almost everywhere equal to $m(f, g, h)$, so that $\mathcal{L}^{1}(X, \mu)$ is a median pseudo-metric space. We conclude that $L^{1}(X, \mu)$ is median because it is the metric quotient of $\mathcal{L}^{1}(X, \mu)$.

(7) (symmetric differences) Let $(X, \mathcal{B}, \mu)$ still denote a measured space. For any subset $A \subset X$, we define

$$
\mathcal{B}_{A}=\{B \subseteq X \mid A \triangle B \in \mathcal{B}, \mu(A \triangle B)<+\infty\} .
$$

Denote as usual by $\chi_{C}$ the characteristic function of a set $C$. Then the map $\chi^{A}: \mathcal{B}_{A} \rightarrow \mathcal{L}^{1}(X, \mu)$ defined by $B \mapsto \chi_{A \triangle B}$ is injective. The range of $\chi^{A}$ consists in the class $\mathcal{S}^{1}(X, \mu)$ of all characteristic functions of measurable subsets with finite measure. Indeed the preimage of $\chi_{B^{\prime}}$ (with $B^{\prime} \in \mathcal{B}, \mu\left(B^{\prime}\right)<+\infty$ ) is the 
subset $B:=A \triangle B^{\prime}$. Observe that the $L^{1}$-pseudo-distance between two functions $\chi_{B^{\prime}}$ and $\chi_{C^{\prime}}$ in $\mathcal{S}^{1}(X, \mu)$ is equal to $\mu\left(B^{\prime} \triangle C^{\prime}\right)$. Since we have

$$
(A \triangle B) \triangle(A \triangle C)=B \triangle C,
$$

it follows that for any two elements $B_{1}, B_{2} \in \mathcal{B}_{A}$ the symmetric difference $B_{1} \triangle B_{2}$ is measurable with finite measure, and the pull-back of the $L^{1}$-pseudodistance by the bijection $\mathcal{B}_{A} \rightarrow \mathcal{S}^{1}(X, \mu)$ is the pseudo-metric pdist ${ }_{\mu}$ defined by $\operatorname{pdist}_{\mu}\left(B_{1}, B_{2}\right)=\mu\left(B_{1} \triangle B_{2}\right)$.

We claim that $\left(\mathcal{B}_{A}\right.$, pdist $\left._{\mu}\right)$ is a median pseudo-metric space, or equivalently that $\mathcal{S}^{1}(X, \mu)$ is a median subspace of $\mathcal{L}^{1}(X, \mu)$. This follows easily from the explicit formula:

$$
m\left(\chi_{A}, \chi_{B}, \chi_{C}\right)=\chi_{(A \cup B) \cap(A \cup C) \cap(B \cup C)}=\chi_{(A \cap B) \cup(A \cap C) \cup(B \cap C)} .
$$

Note that $I\left(\chi_{A}, \chi_{B}\right) \cap \mathcal{S}^{1}(X, \mu)$ is composed of the characteristic functions $\chi_{C}$ such that there exists $C^{\prime} \in \mathcal{B}$ satisfying $\mu\left(C^{\prime} \triangle C\right)=0$ and $A \cap B \subset C^{\prime} \subset A \cup B$. Later we will prove that any median space embeds isometrically as a median subspace of some space $\mathcal{S}^{1}(X, \mu)$ (compare with the similar result in the context of median algebras appearing in Corollary 4.11).

Remark 2.9. In view of Lemma 3.13 and of Example 2.8, (6), a metric space ( $X$, dist) is submedian if and only if it embeds isometrically in a space $L^{1}(\mathcal{W}, \mu)$, for some measured space $(\mathcal{W}, \mu)$. Thus, the notion of submedian space coincides with the notion of metric space of type 1 as defined in [BCK66, Troisième partie, §2]. Similarly, a submedian metric is the same thing as a metric of type 1 .

Remark 2.10. (1) It is not possible in general to define for every submedian space $Y$ a median completion, that is a median space containing an isometric copy of $Y$, and such that any isometric embedding of $Y$ into a median space extends to it. This can be seen in the following example.

Let $E=\mathbb{R}^{7}$ endowed with the $\ell_{1}$ norm, and let $\left\{e_{i} ; i=1,2, \ldots, 7\right\}$ be the canonical basis. Let $Y_{x}$ be the set composed of the four points $A, B, C, D$ in $E$ defined by $A=$ $\frac{x}{2}\left(e_{1}+e_{2}+e_{3}\right)+(1-x) e_{4}, B=\frac{x}{2}\left(-e_{1}-e_{2}+e_{3}\right)+(1-x) e_{5}, C=\frac{x}{2}\left(e_{1}-e_{2}-e_{3}\right)+(1-x) e_{6}$, $D=\frac{x}{2}\left(-e_{1}+e_{2}-e_{3}\right)+(1-x) e_{7}$, where $x \in[0,1]$.

Any two distinct points in $Y_{x}$ are at $\ell_{1}$-distance 2 . Thus all $Y_{x}$ with the $\ell_{1}$-distance are pairwise isometric. The median hull of $Y_{x}$ is composed of $Y_{x}$ itself and of the eight vertices of a cube of edge length $x$ defined by $\frac{x}{2}\left( \pm e_{1} \pm e_{2} \pm e_{3}\right)$. Thus, for two distinct values $x \neq x^{\prime}$ the median hulls of $Y_{x}$ and of $Y_{x^{\prime}}$ are not isometric.

Note that the median hull of $Y_{0}$ is the simplicial tree with five vertices, four of which are endpoints. The median hull of $Y_{1}$ is the set of eight vertices of the unit cube. Consequently, it cannot even be guaranteed that two median hulls of two isometric submedian spaces are isomorphic as median algebras. 
(2) Given a subspace $Y$ of a median space $X$, it is in general not possible to extend an isometry of $Y$ to an isometry (or at least an isomorphisms of median algebras) of the median hull of $Y$.

With the same notations as in (1), the isometry $Y_{0} \times Y_{1} \rightarrow Y_{0} \times Y_{1}$ switching the points of $Y_{0}$ with the points of $Y_{1}$ cannot be extended to the median hull of $Y_{0} \times Y_{1}$ in $E \times E$.

\subsection{Convexity and gate property in median spaces.}

Definition 2.11. Let ( $X$, pdist) denote some pseudo-metric space. A subset $Y \subset X$ is said to be convex if for any $a, b \in Y$ the set $I(a, b)$ is contained in $Y$. It is quasi-convex if for any $a, b \in Y$ the set $I(a, b)$ is contained in $\overline{\mathcal{N}}_{M}(Y)$ for some $M$ uniform in $a, b \in Y$. The convex hull of a subset $Y \subset X$ is the intersection of all convex subsets containing $Y$.

Note that any convex subspace of a median space is median but not the converse, as for instance any subset of cardinality two is a median subspace, while it might not be convex. The median hull of a subset is contained in the convex hull, and as the example above shows the inclusion may be strict.

We now introduce a notion which is related to convexity in median spaces, and which is commonly used in the theory of Tits buildings (see for example [Sch85]).

Definition 2.12 (gate). Let ( $X$, dist) be a metric space, let $Y$ be a subset of $X$, and $x$ some point in $X$.

We say that a point $p \in X$ is between $x$ and $Y$ if it is between $x$ and any $y \in Y$. When a point $p \in Y$ is between $x$ and $Y$, we say that $p$ is a gate between $x$ and $Y$. Note that there is always at most one gate $p$ between $x$ and $Y$, and that $\operatorname{dist}(x, p)=\operatorname{dist}(x, Y)$.

We say that $Y$ is gate-convex if for any point $x \in X$ there exists a gate (in $Y$ ) between $x$ and $Y$. We then denote by $\pi_{Y}(x)$ this gate, and call the map $\pi_{Y}$ the projection map onto $Y$.

Lemma 2.13 (gate-convex subsets). (1) The projection map onto a gate-convex subset is 1-Lipschitz.

(2) Any gate-convex subset is closed and convex.

(3) In a complete median space, any closed convex subset is gate-convex.

In other words, for closed subsets of a complete median space, convexity is equivalent to gate-convexity.

Proof. (1) Let $x, x^{\prime}$ be two points in a metric space $X$, and let $p, p^{\prime}$ be the respective gates between $x, x^{\prime}$ and a gate-convex subset $Y$. Since $\left(x, p, p^{\prime}\right)$ and $\left(x^{\prime}, p^{\prime}, p\right)$ are geodesic sequences, we have that

$$
\begin{aligned}
\operatorname{dist}(x, p)+\operatorname{dist}\left(p, p^{\prime}\right) & \leq \operatorname{dist}\left(x, x^{\prime}\right)+\operatorname{dist}\left(x^{\prime}, p^{\prime}\right) \\
\operatorname{dist}\left(x^{\prime}, p^{\prime}\right)+\operatorname{dist}\left(p^{\prime}, p\right) & \leq \operatorname{dist}\left(x^{\prime}, x\right)+\operatorname{dist}(x, p)
\end{aligned}
$$

By summing up the two inequalities, we conclude that $\operatorname{dist}\left(p, p^{\prime}\right) \leq \operatorname{dist}\left(x, x^{\prime}\right)$. 
(2) Assume that $Y$ is gate-convex and that $(x, y, z)$ is a geodesic sequence with $x, z \in$ $Y$. Let $p$ be the gate between $y$ and $Y$, meaning that $(y, p, x)$ and $(y, p, z)$ are geodesic sequences. Hence $(x, p, y, p, z)$ is a geodesic sequence, which forces $y=p \in Y$.

Any point $x$ in the closure of $Y$ satisfies $\operatorname{dist}(x, Y)=0$. Thus if $p$ is the gate between $x$ and $Y$ we have $\operatorname{dist}(x, p)=0$, hence $x \in Y$. We conclude that $Y$ is closed.

(3) Let $Y$ be a closed convex subset of a complete median space $X$. For any $x \in X$ choose a sequence $\left(y_{k}\right)_{k \geq 0}$ of points in $Y$ such that $\operatorname{dist}\left(y_{k}, x\right)$ tends to $\operatorname{dist}(x, Y)$. First observe that $\left(y_{k}\right)_{k \geq 0}$ is a Cauchy sequence. Indeed, denote by $\epsilon_{k}=\operatorname{dist}\left(y_{k}, x\right)-\operatorname{dist}(Y, x)$, which clearly is a sequence of positive numbers converging to zero. Let $m_{k, \ell}$ be the median point of $\left(x, y_{k}, y_{\ell}\right)$. Then $\operatorname{dist}\left(x, y_{k}\right)+\operatorname{dist}\left(x, y_{\ell}\right)=2 \operatorname{dist}\left(x, m_{k, \ell}\right)+\operatorname{dist}\left(y_{k}, y_{\ell}\right)$ and so by convexity of $Y$ we have $\operatorname{dist}\left(x, y_{k}\right)+\operatorname{dist}\left(x, y_{\ell}\right) \geq 2 \operatorname{dist}(x, Y)+\operatorname{dist}\left(y_{k}, y_{\ell}\right)$. It follows that $\operatorname{dist}\left(y_{k}, y_{\ell}\right) \leq \epsilon_{k}+\epsilon_{\ell}$. Since $X$ is complete the sequence $\left(y_{k}\right)_{k \geq 0}$ has a limit $p$ in $X$. Since $Y$ is closed, the point $p$ is in $Y$. Note that $\operatorname{dist}(x, p)=\operatorname{dist}(x, Y)$. It remains to check that $p$ is between $x$ and $Y$.

Let $y$ be some point in $Y$, and let $m$ be the median point of $x, p, y$. By convexity of $Y$ we have $m \in Y$, so that $\operatorname{dist}(x, m) \geq \operatorname{dist}(x, Y)$. We also have $\operatorname{dist}(x, p)=\operatorname{dist}(x, m)+$ $\operatorname{dist}(m, p)$. Since $\operatorname{dist}(x, p)=\operatorname{dist}(x, Y)$ we get $\operatorname{dist}(m, p)=0$ as desired.

We now prove that in a median space the metric intervals are gate-convex.

Lemma 2.14. In a median metric space any interval $I(a, b)$ is gate-convex, and the gate between an arbitrary point $x$ and $I(a, b)$ is $m(x, a, b)$.

Proof. Consider an arbitrary point $x$ in the ambient median metric space $X, p$ the median point $m(x, a, b)$ and $y$ an arbitrary point in $I(a, b)$. We will show that $(x, p, y)$ is a geodesic sequence.

We consider the median points $a^{\prime}=m(x, a, y), b^{\prime}=m(x, b, y)$ and $p^{\prime}=m\left(x, a^{\prime}, b^{\prime}\right)$. Note that $p^{\prime} \in I\left(x, a^{\prime}\right) \subset I(x, a)$ and similarly $p^{\prime} \in I(x, b)$.

Since $(a, y, b),\left(a, a^{\prime}, y\right)$ and $\left(y, b^{\prime}, b\right)$ are geodesic sequences, the sequence $\left(a, a^{\prime}, y, b^{\prime}, b\right)$ is geodesic as well. So $I\left(a^{\prime}, b^{\prime}\right) \subset I(a, b)$, hence $p^{\prime} \in I(a, b)$.

We proved that $p^{\prime} \in I(x, a) \cap I(x, b) \cap I(a, b)$, which by the uniqueness of the median point implies $p^{\prime}=p$. It follows that $p \in I\left(x, a^{\prime}\right) \subset I(x, y)$.

We can now deduce that the median map is 1-Lipschitz, in each variable and on $X \times X \times X$ endowed with the $\ell_{1}$ metric.

Corollary 2.15. Let $X$ be a median space.

(1) For any two fixed points $a, b \in X$ the interval $I(a, b)$ is closed and convex, and the map $x \mapsto m(x, a, b)$ is 1-Lipschitz.

(2) The median map $m: X \times X \times X \rightarrow X$ is 1-Lipschitz (here $X \times X \times X$ is endowed with the $\ell_{1}$-product metric as defined in Example 2.8, (1)).

Proof. Combine Lemma 2.14 and Lemma 2.13, and use the fact that, given six points $a, b, c, a^{\prime}, b^{\prime}, c^{\prime} \in X$, the distance between the median points $m(a, b, c)$ and $m\left(a^{\prime}, b^{\prime}, c^{\prime}\right)$ is 
at most

$$
\operatorname{dist}\left(m(a, b, c), m\left(a^{\prime}, b, c\right)\right)+\operatorname{dist}\left(m\left(a^{\prime}, b, c\right), m\left(a^{\prime}, b^{\prime}, c\right)\right)+\operatorname{dist}\left(m\left(a^{\prime}, b^{\prime}, c\right), m\left(a^{\prime}, b^{\prime}, c^{\prime}\right)\right) .
$$

2.3. Approximate geodesics and medians; completions of median spaces. We prove that the median property is preserved under metric completion. In order to do it, we need an intermediate result stating that in a median space, approximate geodesics are close to geodesics, and approximate medians are close to medians. We begin by defining approximate geodesics and medians.

Definition 2.16. Let ( $X$, dist) be a metric space and let $\delta$ be a non-negative real number. We say that $z$ is between $x$ and $y$ up to $\delta$ provided

$$
\operatorname{dist}(x, z)+\operatorname{dist}(z, y) \leq \operatorname{dist}(x, y)+\delta .
$$

We say that $\left(a_{1}, a_{2}, \ldots, a_{n}\right)$ is a $\delta$-geodesic sequence if

$$
\operatorname{dist}\left(a_{1}, a_{2}\right)+\operatorname{dist}\left(a_{2}, a_{3}\right)+\cdots+\operatorname{dist}\left(a_{n-1}, a_{n}\right) \leq \operatorname{dist}\left(a_{1}, a_{n}\right)+\delta .
$$

Notation 2.17. Let $x, y$ be two points of $X$. We denote by $I_{\delta}(a, b)$ the set of points that are between $a$ and $b$ up to $\delta$.

Let $x, y, z$ be three points of $X$. We denote the intersection $I_{2 \delta}(a, b) \cap I_{2 \delta}(b, c) \cap I_{2 \delta}(a, c)$ by $M_{\delta}(a, b, c)$.

In accordance with the previous notation whenever $\delta=0$ the index is dropped.

Lemma 2.18. Given $\delta, \delta^{\prime} \geq 0$ for every $c \in I_{\delta}(a, b)$ the set $I_{\delta^{\prime}}(a, c) \subset I_{\delta+\delta^{\prime}}(a, b)$.

Definition 2.19. Let $x, y, z$ denote three points in a metric space. If $M_{\delta}(x, y, z)$ is non-empty then any point in it is called a $\delta$-median point for $x, y, z$.

Lemma 2.20. Let ( $X$, dist) be a median space, and $a, b, c$ three arbitrary points in it.

(i) The set $I_{2 \delta}(a, b)$ coincides with $\overline{\mathcal{N}}_{\delta}(I(a, b))$.

(ii) The following sequence of inclusions holds

$$
\bar{B}(m(a, b, c), \delta) \subseteq M_{\delta}(a, b, c) \subseteq \bar{B}(m(a, b, c), 3 \delta) .
$$

Proof. Statement (i) immediately follows from Lemma 2.14 .

The first inclusion in (1) is obvious. We prove the second inclusion. To do it, we consider the median points $p_{1}=m(p, a, b), p_{2}=m(p, b, c), p_{3}=m(p, a, c), q=$ $m\left(p_{1}, b, c\right), r=m(q, a, c)$.

First we show that $r=m(a, b, c)$. Indeed $r \in I(a, c)$ by definition. We also have $r \in I(q, c)$, and since $q \in I(c, b)$ it follows that $r \in I(b, c)$. Finally we have $r \in I(a, q)$. Now $q \in I\left(p_{1}, b\right)$ and $p_{1} \in I(a, b)$, so $q \in I(a, b)$. It follows that $r \in I(a, b)$.

It remains to estimate the distance between $p$ and $r$. According to (i) and Lemma 2.14 the point $p$ is at distance at most $\delta$ from $p_{1}, p_{2}$ and $p_{3}$ respectively. 
By Corollary 2.15 we have $\operatorname{dist}\left(p_{2}, q\right) \leq \operatorname{dist}\left(p, p_{1}\right) \leq \delta$. Hence $\operatorname{dist}(p, q) \leq 2 \delta$. Applying Corollary 2.15 again we get $\operatorname{dist}\left(p_{3}, r\right) \leq \operatorname{dist}(p, q) \leq 2 \delta$, consequently $\operatorname{dist}(p, r) \leq$ $3 \delta$.

Proposition 2.21. Let (X, dist) be a median space, let $X \rightarrow \hat{X}$ be the metric completion, and let dist be the distance on $\hat{X}$. Then $(\hat{X}$, dist) is a median space as well (and $X$ is a dense median subspace).

Proof. The median map $m: X \times X \times X \rightarrow X \subset \hat{X}$ is 1-Lipschitz by Corollary 2.15. Thus it extends to a 1-Lipschitz map $\hat{X} \times \hat{X} \times \hat{X} \rightarrow \hat{X}$, also denoted by $m$.

Clearly for any three points $a, b, c$ in $\hat{X}$, the point $m(a, b, c)$ is median for $a, b, c$. We now prove that $m(a, b, c)$ is the unique median point for $a, b, c$. Let $p$ be another median point for $a, b, c$. The points $a, b, c$ are limits of sequences $\left(a_{n}\right),\left(b_{n}\right),\left(c_{n}\right)$ of points in $X$. Let $m_{n}$ be the median point of $a_{n}, b_{n}, c_{n}$. Set $\delta_{n}=\operatorname{dist}\left(a, a_{n}\right)+\operatorname{dist}\left(b, b_{n}\right)+\operatorname{dist}\left(c, c_{n}\right)$.

We show that $p$ is a $\delta_{n}$-median point for $a_{n}, b_{n}, c_{n}$. Indeed we have that $\operatorname{dist}\left(a_{n}, p\right)+$ $\operatorname{dist}\left(p, b_{n}\right)$ is at most $\operatorname{dist}\left(a_{n}, a\right)+\operatorname{dist}(a, p)+\operatorname{dist}(p, b)+\operatorname{dist}\left(b, b_{n}\right)=\operatorname{dist}\left(a_{n}, a\right)+$ $\operatorname{dist}(a, b)+\operatorname{dist}\left(b, b_{n}\right) \leq 2 \operatorname{dist}\left(a, a_{n}\right)+\operatorname{dist}\left(a_{n}, b_{n}\right)+2 \operatorname{dist}\left(b, b_{n}\right) \leq \operatorname{dist}\left(a_{n}, b_{n}\right)+2 \delta_{n}$. The other inequalities are proved similarly.

The point $p$ is also the limit of a sequence of points $p_{n}$ in $X$, $\operatorname{such}$ that $\operatorname{dist}\left(p, p_{n}\right) \leq \delta_{n}$. It follows that $p_{n}$ is a $2 \delta_{n}$-median point for $a_{n}, b_{n}, c_{n}$. By Lemma 2.20 we then have that $\operatorname{dist}\left(p_{n}, m_{n}\right) \leq 6 \delta_{n}$. Since $\delta_{n} \rightarrow 0$ we get $p=m(a, b, c)$.

2.4. Rectangles and parallel pairs. In a median space $X$, the following notion of rectangle will allow us to treat median spaces as a continuous version of the 1-skeleton of a $\mathrm{CAT}(0)$ cube complex.

Definition 2.22. A rectangle in a metric space ( $X$, dist) is a sequence $[a, b, c, d]$ such that the four sequences $(a, b, c),(b, c, d),(c, d, a)$ and $(d, a, b)$ are geodesic.

Remark 2.23. (1) We use the square brackets in order to avoid confusion between geodesic sequences and rectangles.

(2) By the triangle inequality, a rectangle $[a, b, c, d]$ has the following properties: $\operatorname{dist}(a, b)=\operatorname{dist}(c, d), \operatorname{dist}(a, d)=\operatorname{dist}(b, c)$ and $\operatorname{dist}(a, c)=\operatorname{dist}(b, d)$.

(3) (rectangles in intervals) If $x, y \in I(a, b)$ then $[x, m(x, y, a), y, m(x, y, b)]$ is a rectangle.

(4) (subdivision of rectangles) Let $[a, b, c, d]$ be a rectangle. Let $e \in I(a, d)$ and $f=m(e, b, c)$. Then $[a, b, f, e]$ and $[c, d, e, f]$ are rectangles.

Definition 2.24. (parallelism on pairs) Two pairs $(a, b)$ and $(d, c)$ are parallel if $[a, b, c, d]$ is a rectangle.

We mention without proof the following remarkable fact that confirms the analogy with $\mathrm{CAT}(0)$ cube complexes:

Proposition 2.25. In a median space the parallelism on pairs is an equivalence relation. 
We now explain how to any 4-tuple of points one can associate a rectangle.

Lemma 2.26. Let $x, y$ and $a, b$ be two pairs of points in a median space. Then there exists a unique rectangle $\left[a^{\prime}, x^{\prime}, b^{\prime}, y^{\prime}\right]$ satisfying the following properties:

(1) the following sequences are geodesic:

$$
\left(a, a^{\prime}, x^{\prime}, x\right),\left(x, x^{\prime}, b^{\prime}, b\right),\left(b, b^{\prime}, y^{\prime}, y\right),\left(y, y^{\prime}, a^{\prime}, a\right) ;
$$

(2) $\left(x, x^{\prime}, y^{\prime}, y\right)$ is a geodesic sequence;

(3) $\left(a, a^{\prime}, b^{\prime}\right)$ and $\left(b, b^{\prime}, a^{\prime}\right)$ are geodesic sequences.

Proof of Lemma 2.26. Existence. Let $a^{\prime}=m(a, x, y)$ and $b^{\prime}=m(b, x, y)$, and let $x^{\prime}=$ $m\left(x, a^{\prime}, b^{\prime}\right)$ and $y^{\prime}=m\left(y, a^{\prime}, b^{\prime}\right)$ (see Figure 1). Then $\left[a^{\prime}, x^{\prime}, b^{\prime}, y^{\prime}\right]$ is a rectangle by Remark 2.23, (3). Properties (1) and (2) follow immediately from the construction, property (3) follows from Lemma 2.14 applied to $a$ and $b^{\prime} \in I(x, y)$, respectively to $b$ and $a^{\prime} \in I(x, y)$.

Unicity. Let $\left[a^{\prime}, x^{\prime}, b^{\prime}, y^{\prime}\right]$ be a rectangle satisfying the three required properties. Properties (1), (2) and the fact that $\left[a^{\prime}, x^{\prime}, b^{\prime}, y^{\prime}\right]$ is a rectangle imply that $a^{\prime}=m(a, x, y)$ and $b^{\prime}=m(b, x, y)$. Again property $(2)$ and the fact that $\left[a^{\prime}, x^{\prime}, b^{\prime}, y^{\prime}\right]$ is a rectangle imply that $x^{\prime}=m\left(x, a^{\prime}, b^{\prime}\right)$ and $y^{\prime}=m\left(y, a^{\prime}, b^{\prime}\right)$.

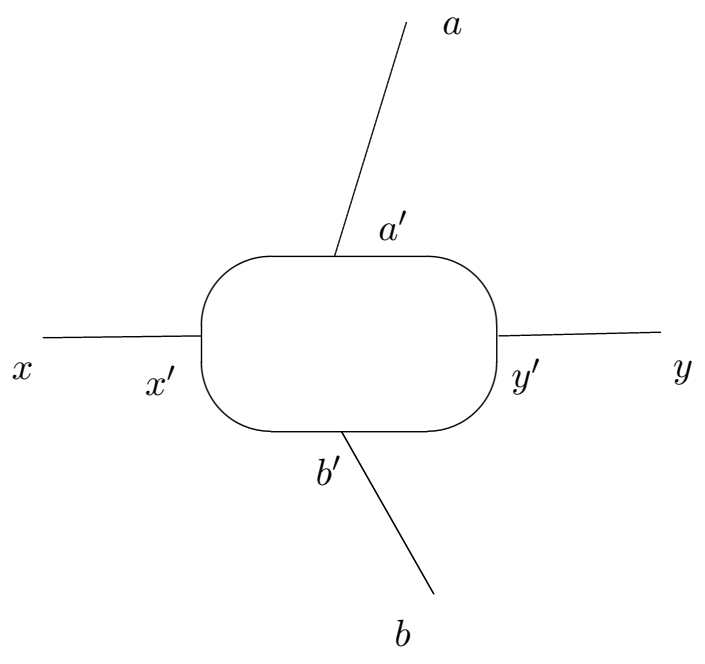

Figure 1. Central rectangle.

Definition 2.27. We call $\left[a^{\prime}, x^{\prime}, b^{\prime}, y^{\prime}\right]$ as in Lemma 2.26 the central rectangle of the four points $x, y, a, b$. 
Remark 2.28. Condition (3) cannot be improved to " $\left(a, a^{\prime}, b^{\prime}, b\right)$ is a geodesic sequence", as shown by the example of a unit cube in $\mathbb{R}^{3}$ with $x, y$ two opposite vertices of the lower horizontal face, and $a, b$ the two opposite vertices of the upper horizontal face that are not above $x$ and $y$ (see Figure 2).

The order between the two pairs $x, y$ and $a, b$ is therefore important in determining the central rectangle, since the conditions (2) and (3) show that the definition is not symmetric in $(x, y)$ and $(a, b)$. In some sense, in the example above one has the choice as to what would be the central rectangle of the 4 -tuple $x, y, a, b$ between the lower horizontal face and the upper horizontal face. It is one or the other, depending on the order in which the pairs $x, y$ and $a, b$ are considered.

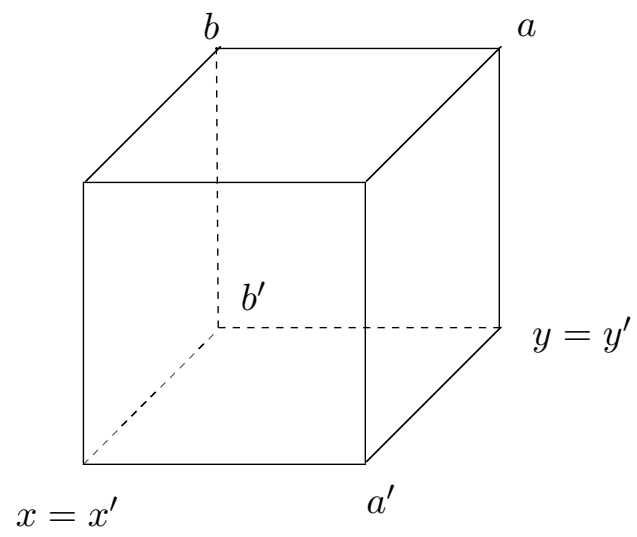

Figure 2. Example of central rectangle.

Property (3) in Lemma 2.26 can be slightly improved as follows.

Lemma 2.29. Let $a, b, a^{\prime}, b^{\prime}$ be four points such that $\left(a, a^{\prime}, b^{\prime}\right)$ and $\left(b, b^{\prime}, a^{\prime}\right)$ are geodesic sequences. Then there exists a geodesic sequence $\left(a, a^{\prime \prime}, b^{\prime \prime}, b\right)$ such that $\left(a^{\prime \prime}, b^{\prime \prime}\right)$ and $\left(a^{\prime}, b^{\prime}\right)$ are parallel.

Proof. Let $b^{\prime \prime}=m\left(a, b, b^{\prime}\right)$ and $a^{\prime \prime}=m\left(a, a^{\prime}, b^{\prime \prime}\right)$. Then $\left(a, a^{\prime}, b^{\prime}\right)$ and $\left(b, b^{\prime}, a^{\prime}\right)$ geodesic imply $\left(a^{\prime \prime}, a^{\prime}, b^{\prime}\right)$ and $\left(b^{\prime \prime}, b^{\prime}, a^{\prime}\right)$ geodesic. Also $\left(a, a^{\prime \prime}, b^{\prime \prime}, b^{\prime}\right)$ is geodesic by construction, and so is $\left(a^{\prime}, a^{\prime \prime}, b^{\prime \prime}\right)$. Thus $\left[a^{\prime}, b^{\prime}, b^{\prime \prime}, a^{\prime \prime}\right]$ is a rectangle.

Also by construction $\left(a, a^{\prime \prime}, b^{\prime \prime}, b\right)$ is a geodesic. 


\section{The median space associated to a space with measured Walls.}

3.1. Preliminaries on measures. We recall the relevant definitions on measured spaces. A reference is [Bau01], whose terminology we adopt here. Let $Y$ be a non-empty set and let $\mathcal{P}(Y)$ be the power set of $Y$. A ring is a subset $\mathcal{R}$ of $\mathcal{P}(Y)$ such that:

(1) $\emptyset \in \mathcal{R}$.

(2) If $A, B \in \mathcal{R}$ then $A \cup B \in \mathcal{R}$.

(3) If $A, B \in \mathcal{R}$ then $A \backslash B \in \mathcal{R}$.

A $\sigma$-algebra is a subset $\mathcal{B}$ in $\mathcal{P}(Y)$ such that

(1) $\emptyset \in \mathcal{A}$.

(2) If $A_{n} \in \mathcal{B}, n \in \mathbb{N}$, then $\bigcup_{n \in \mathbb{N}} A_{n} \in \mathcal{B}$.

(3) If $A \in \mathcal{A}$ then $A^{c} \in \mathcal{A}$.

Given a ring $\mathcal{R}$, a premeasure on it is a function $\mu: \mathcal{R} \rightarrow[0,+\infty]$ such that $\left(M_{0}\right) \mu(\emptyset)=0$;

$\left(M_{1}\right)$ for any sequence of pairwise disjoint sets $\left(A_{n}\right)_{n \in \mathbb{N}}$ in $\mathcal{R}$ such that $\bigsqcup_{n \in \mathbb{N}} A_{n} \in \mathcal{R}$,

$$
\mu\left(\bigsqcup_{n \in \mathbb{N}} A_{n}\right)=\sum_{n \in \mathbb{N}} \mu\left(A_{n}\right) .
$$

Property $\left(M_{1}\right)$ is equivalent to

$\left(M_{1}^{\prime}\right) \mu(A \sqcup B)=\mu(A)+\mu(B)$;

$\left(M_{1}^{\prime \prime}\right)$ If $\left(A_{n}\right)_{n \in \mathbb{N}}$ is a non-increasing sequence of sets in $\mathcal{R}$ such that $\bigcap_{n \in \mathbb{N}} A_{n}=\emptyset$, then $\lim _{n \rightarrow \infty} \mu\left(A_{n}\right)=0$.

A premeasure is called $\sigma$-finite if there exists a sequence $\left(A_{n}\right)$ in $\mathcal{R}$ such that $\mu\left(A_{n}\right)<$ $+\infty$ for every $n$, and $\bigcup_{n} A_{n}=Y$.

A premeasure defined on a $\sigma$-algebra is called a measure.

We need a precise version of Caratheodory's Theorem on the extension of any premeasure to a measure, therefore we recall here the notion of outer measure. For every $Q \subset Y$ let $\mathcal{U}(Q)$ designate the set of all sequences $\left(A_{n}\right)$ in $\mathcal{R}$ such that $Q \subset \bigcup_{n} A_{n}$. Define $\mu^{*}(Q)=+\infty$ if $\mathcal{U}(Q)=\emptyset$; if $\mathcal{U}(Q) \neq \emptyset$ then

$$
\mu^{*}(Q)=\inf \left\{\sum_{n=1}^{\infty} \mu\left(A_{n}\right) ;\left(A_{n}\right) \in \mathcal{U}(Q)\right\} .
$$

The function $\mu^{*}$ is an outer measure on the set $Y$.

A subset $A$ of $Y$ is called $\mu^{*}$-measurable if for every $Q \in \mathcal{P}(Y)$,

$$
\mu^{*}(Q)=\mu^{*}(Q \cap A)+\mu^{*}\left(Q \cap A^{c}\right) .
$$

Theorem 3.1 (Carathéodory [Bau01], $§ I .5) . \quad$ (1) The collection $\mathcal{A}^{*}$ of $\mu^{*}$-measurable sets is a $\sigma$-algebra containing $\mathcal{R}$, and the restriction of $\mu^{*}$ to $\mathcal{A}^{*}$ is a measure, while the restriction of $\mu^{*}$ to $\mathcal{R}$ coincides with $\mu$. 
(2) If $\mu$ is $\sigma$-finite, then it has a unique extension to a measure on the $\sigma$-algebra generated by $\mathcal{R}$.

3.2. Spaces with measured walls. From [HP98], we recall that a wall of a set $X$ is a partition $X=h \sqcup h^{c}$ (where $h$ is possibly empty or the whole $X$ ). A collection $\mathcal{H}$ of subsets of $X$ is called a collection of half-spaces if for every $h \in \mathcal{H}$ the complementary subset $h^{c}$ is also in $\mathcal{H}$. We call collection of walls on $X$ the collection $\mathcal{W}_{\mathcal{H}}$ of pairs $w=\left\{h, h^{c}\right\}$ with $h \in \mathcal{H}$. For a wall $w=\left\{h, h^{c}\right\}$ we call $h$ and $h^{c}$ the two half-spaces bounding $w$.

We say that a wall $w=\left\{h, h^{c}\right\}$ separates two disjoint subsets $A, B$ in $X$ if $A \subset h$ and $B \subset h^{c}$ or vice-versa and denote by $\mathcal{W}(A \mid B)$ the set of walls separating $A$ and $B$. In particular $\mathcal{W}(A \mid \emptyset)$ is the set of walls $w=\left\{h, h^{c}\right\}$ such that $A \subset h$ or $A \subset h^{c}$; hence $\mathcal{W}(\emptyset \mid \emptyset)=\mathcal{W}$.

When $A=\left\{x_{1}, \ldots, x_{n}\right\}, B=\left\{y_{1}, \ldots, y_{m}\right\}$ we write

$$
\mathcal{W}(A \mid B)=\mathcal{W}\left(x_{1}, \ldots, x_{n} \mid y_{1}, \ldots, y_{m}\right) .
$$

We use the notation $\mathcal{W}(x \mid y)$ to designate $\mathcal{W}(\{x\} \mid\{y\})$. We call any set of walls of the form $\mathcal{W}(x \mid y)$ a wall-interval. By convention $\mathcal{W}(A \mid A)=\emptyset$ for every non-empty set $A$.

Definition 3.2 (space with measured walls [CMV04]). A space with measured walls is a 4-uple $(X, \mathcal{W}, \mathcal{B}, \mu)$, where $\mathcal{W}$ is a collection of walls, $\mathcal{B}$ is a $\sigma$-algebra of subsets in $\mathcal{W}$ and $\mu$ is a measure on $\mathcal{B}$, such that for every two points $x, y \in X$ the set of separating walls $\mathcal{W}(x \mid y)$ is in $\mathcal{B}$ and it has finite measure. We denote by $\operatorname{pdist}_{\mu}$ the pseudo-metric on $X$ defined by $\operatorname{pdist}_{\mu}(x, y)=\mu(\mathcal{W}(x \mid y))$, and we call it the wall pseudo-metric.

Convention 3.3. We allow for the possibility that $\mathcal{W}$ contains the trivial wall $\{X, \emptyset\}$.

Lemma 3.4. The collection $\mathcal{R}$ of disjoint unions $\bigsqcup_{i=1}^{n} \mathcal{W}\left(F_{i} \mid G_{i}\right)$, where $n \in \mathbb{N}^{*}$, and $F_{i}, G_{i}$ are finite non-empty sets for every $i=1,2, \ldots, n$, is a ring.

Proof. Property (1) is obviously satisfied.

We first note that $\mathcal{W}(F \mid G) \cap \mathcal{W}\left(F^{\prime} \mid G^{\prime}\right)=\mathcal{W}\left(F \cup F^{\prime} \mid G \cup G^{\prime}\right) \sqcup \mathcal{W}\left(F \cup G^{\prime} \mid G \cup F^{\prime}\right)$.

Let now $F, G$ be two finite non-empty sets, and let $\mathcal{S}$ be the set of pairs $\left(S_{1}, S_{2}\right)$ such that $S_{1} \subseteq F, S_{2} \subseteq G$ and $S_{1} \cup S_{2} \subsetneq F \cup G$. It is easily seen that

$$
\mathcal{W}(F \mid G)^{c}=\bigsqcup_{\left(S_{1}, S_{2}\right) \in \mathcal{S}} \mathcal{W}\left(S_{1} \cup S_{2} \mid(F \cup G) \backslash\left(S_{1} \cup S_{2}\right)\right)
$$

From these two statements it follows that $\mathcal{R}$ satisfies property (3), i.e. it is closed with respect to the operation $\backslash$. This and the fact that $\mathcal{R}$ is closed with respect to intersection implies that $\mathcal{R}$ is closed with respect to union.

Theorem 3.1 and Lemma 3.4 imply the following.

Proposition 3.5 (minimal data required for a structure of measured walls). Let $X$ be $a$ space and let $\mathcal{W}$ be a collection of walls on it. A structure of measured walls can be defined on $(X, \mathcal{W})$ if and only if on the ring $\mathcal{R}$ composed of disjoint unions $\bigsqcup_{i=1}^{n} \mathcal{W}\left(F_{i} \mid G_{i}\right)$, where 
$n \in \mathbb{N}^{*}$, and $F_{i}, G_{i}, i=1,2, \ldots, n$, are finite non-empty sets, can be defined a premeasure $\mu$ such that for every $x, y \in X, \mu(\mathcal{W}(x \mid y))$ is finite.

Let $(X, \mathcal{W}, \mathcal{B}, \mu)$ and $\left(X^{\prime}, \mathcal{W}^{\prime}, \mathcal{B}^{\prime}, \mu^{\prime}\right)$ be two spaces with measured walls, and let $\phi$ : $X \rightarrow X^{\prime}$ be a map.

Definition 3.6. The map $\phi$ is a homomorphism between spaces with measured walls provided that:

- for any $w^{\prime}=\left\{h^{\prime}, h^{\prime c}\right\} \in \mathcal{W}^{\prime}$ we have $\left\{\phi^{-1}\left(h^{\prime}\right), \phi^{-1}\left(h^{\prime c}\right)\right\} \in \mathcal{W}$ - this latter wall we denote by $\phi^{*}\left(w^{\prime}\right)$;

- the map $\phi^{*}: \mathcal{W}^{\prime} \rightarrow \mathcal{W}$ is surjective and for every $B \in \mathcal{B},\left(\phi^{*}\right)^{-1}(B) \in \mathcal{B}^{\prime}$ and $\mu^{\prime}\left(\left(\phi^{*}\right)^{-1}(B)\right)=\mu(B)$.

Note that $\phi$ induces an isometry of the spaces equipped with the wall pseudo-distances.

Consider the set $\mathcal{H}$ of half-spaces determined by $\mathcal{W}$, and the natural projection map $\mathfrak{p}: \mathcal{H} \rightarrow \mathcal{W}, h \mapsto\left\{h, h^{c}\right\}$. The pre-images of the sets in $\mathcal{B}$ define a $\sigma$-algebra on $\mathcal{H}$, which we denote by $\mathcal{B}^{\mathcal{H}}$; hence on $\mathcal{H}$ can be defined a pull-back measure that we also denote by $\mu$. This allows us to work either in $\mathcal{H}$ or in $\mathcal{W}$.

Definition 3.7 ([CN04], [Nic04]). A section $\mathfrak{s}$ for $\mathfrak{p}$ is called admissible if its image contains together with a half-space $h$ all the half-spaces $h^{\prime}$ containing $h$.

Throughout the paper we identify an admissible section $\mathfrak{s}$ with its image $\sigma=\mathfrak{s}(\mathcal{W})$; with this identification, an admissible section becomes a collection of half-spaces, $\sigma$, such that:

- for every wall $w=\left\{h, h^{c}\right\}$ either $h$ or $h^{c}$ is in $\sigma$, but never both;

- if $h \subset h^{\prime}$ and $h \in \sigma$ then $h^{\prime} \in \sigma$.

For any $x \in X$ we denote by $\mathfrak{s}_{x}$ the section of $\mathfrak{p}$ associating to each wall the half-space bounding it and containing $x$. Obviously it is an admissible section. We denote by $\sigma_{x}$ its image, that is the set of half-spaces $h \in \mathcal{H}$ such that $x \in h$.

Note that $\mathfrak{p}\left(\sigma_{x} \triangle \sigma_{y}\right)=\mathcal{W}(x \mid y)$.

Example 3.8 (real hyperbolic space). For all the discussion below, see [CMV04].

Define the half-spaces of the real hyperbolic space $\mathbb{H}^{n}$ to be closed or open geometric half-spaces, so that the boundary of half-spaces is an isometric copy of $\mathbb{H}^{n-1}$ (a geometric hyperplane of $\mathbb{H}^{n}$ ). Note that the associated set of walls $\mathcal{W}_{\mathbb{H}^{n}}$ is identified with the homogeneous space $S O(n, 1) / S O(n-1,1)$. Since the stabilizer of a hyperplane is unimodular, there is a $S O(n, 1)$-invariant borelian measure $\mu_{\mathbb{H}^{n}}$ on the set of walls. Since the set of walls separating two points has a compact closure it has finite measure and thus $\left(\mathbb{H}^{n}, \mathcal{W}_{\mathbb{H}^{n}}, \mathcal{B}, \mu_{\mathbb{H}^{n}}\right)$ is a space with measured walls. By Crofton's formula (Proposition 3 in [CMV04]) up to multiplying the measure $\mu_{\mathbb{H}^{n}}$ by some positive constant the wall pseudo-metric on $\mathbb{H}^{n}$ is just the usual hyperbolic distance.

The following result also allows to produce many examples. 
Lemma 3.9 (pull back of a space with measured walls). Let $(X, \mathcal{W}, \mathcal{B}, \mu)$ be a space with measured walls, let $S$ be a set and $f: S \rightarrow X$ a map. There exists a pull back structure of space with measured walls $\left(S, \mathcal{W}_{S}, \mathcal{B}_{S}, \mu_{S}\right)$ turning $f$ into a homomorphism. Moreover:

(i) if $S$ is endowed with a pseudo-metric pdist and $f$ is an isometry between ( $S$, pdist) and $\left(X\right.$, pdist $\left._{\mu}\right)$, then the wall pseudo-metric pdist $_{\mu_{S}}$ coincides with the initial pseudo-metric pdist;

(ii) if a group $G$ acts on $S$ by bijective transformations and on $X$ by isomorphisms of space with measured walls, and if $f$ is $G$-equivariant, then $G$ acts on $\left(S, \mathcal{W}_{S}, \mathcal{B}_{S}, \mu_{S}\right)$ by isomorphisms of space with measured walls.

Proof. Define the set of walls $\mathcal{W}_{S}$ on $S$ as the set of walls $\left\{f^{-1}(h), f^{-1}\left(h^{c}\right)\right\}$, where $\left\{h, h^{c}\right\}$ is a wall in $X$. This defines a surjective map $f^{*}: \mathcal{W} \rightarrow \mathcal{W}_{S}$. We then consider the pushforward structure of measured space on $\mathcal{W}_{S}$. This defines a structure of measured space with walls on $S$ such that $f$ is a homomorphism of spaces with measured walls.

(i) It is easily seen that for every $x, y \in S,\left(f^{*}\right)^{-1}\left(\mathcal{W}_{S}(x \mid y)\right)=\mathcal{W}(f(x), f(y))$, hence $\operatorname{pdist}_{\mu_{S}}(x, y)=\operatorname{pdist}_{\mu}(f(x), f(y))=\operatorname{pdist}(x, y)$.

(ii) If $f$ is $G$-equivariant then the whole structure of space with measured walls $\left(S, \mathcal{W}_{S}, \mathcal{B}_{S}, \mu_{S}\right)$ is $G$-equivariant.

Example 3.10 (Finite spaces with measured walls). Given any space with measured walls $(X, \mathcal{W}, \mathcal{B}, \mu)$ and any finite subset $F \subseteq X$, one gets a structure of space with measured walls on $F$ via the pull back structure turning the inclusion $\iota: F \rightarrow X$ into a homomorphism. Explicitely, the walls of $F$ will be given by induced partitions from the walls in $X$, so that $\mu(\mathcal{W}(x \mid y))=\mu(\mathcal{W}(\iota(x) \mid \iota(y)))$.

One of the main interests in actions of groups on spaces with measured walls is given by the following result.

Lemma 3.11 ([CMV04], [dCTV06]). Let $G$ be a group acting by automorphisms on a space with measured walls $(X, \mathcal{W}, \mathcal{B}, \mu)$. Let $p>0$ and let $\pi_{p}$ be the representation of $G$ on $L^{p}\left(\mathcal{H}, \mu_{\mathcal{H}}\right)$.

Then for every $x \in X$, the map $b: G \rightarrow L^{p}\left(\mathcal{H}, \mu_{\mathcal{H}}\right)$ defined by $b(g)=\chi_{\sigma_{g x}}-\chi_{\sigma_{x}}$ is a 1-cocycle in $Z^{1}\left(G, \pi_{p}\right)$. In other words, an action of $G$ on $L^{p}\left(\mathcal{H}, \mu_{\mathcal{H}}\right)$ by affine isometries can be defined by:

$$
g \cdot f=\pi_{p}(g) f+b(g)
$$

Remark 3.12. Recall that for a space $L^{p}(X, \mu)$ with $p \in(0,1),\|f\|_{p}=\left(\int|f|^{p} d \mu\right)^{\frac{1}{p}}$ no longer satisfies the usual triangular inequality, it only satisfies a similar inequality with a multiplicative factor added to the second term. On the other hand, $\|f\|_{p}^{p}$ is no longer a norm, but it does satisfy the triangular inequality, hence it defines a metric [KPR84].

In this paper we consider $L^{p}$-spaces endowed with this metric, for $p \in(0,1)$. 
3.3. Embedding a space with measured walls in a median space. Let $(X, \mathcal{W}, \mathcal{B}, \mu)$ be a space with measured walls.

Let now $x_{0}$ denote some base point in $X$. Recall from Example 2.8, (7), that $\mathcal{B}^{\mathcal{H}} \sigma_{x_{0}}$ denotes the set of $A \subset \mathcal{H}$ s.t. $A \triangle \sigma_{x_{0}} \in \mathcal{B}$ and $\mu\left(A \triangle \sigma_{x_{0}}\right)<+\infty$, and that endowed with the pseudo-metric $\operatorname{pdist}_{\mu}(A, B)=\mu(A \Delta B)$ this set becomes a median pseudo-metric space. The map

$$
\chi^{x_{0}}: \mathcal{B}_{\sigma_{x_{0}}}^{\mathcal{H}} \rightarrow \mathcal{S}^{1}(\mathcal{H}, \mu), \chi^{x_{0}}(A)=\chi_{A \triangle \sigma_{x_{0}}}
$$

is an isometric embedding of $\mathcal{B}^{\mathcal{H}}{ }_{\sigma_{x_{0}}}$ into the median subspace $\mathcal{S}^{1}(\mathcal{H}, \mu) \subset \mathcal{L}^{1}(\mathcal{H}, \mu)$, where $\mathcal{S}^{1}(\mathcal{H}, \mu)=\left\{\chi_{B}, B\right.$ is measurable and $\left.\mu(B)<+\infty\right\}$.

The formula $A \Delta \sigma_{x_{1}}=\left(A \Delta \sigma_{x_{0}}\right) \Delta\left(\sigma_{x_{0}} \Delta \sigma_{x_{1}}\right)$ and the fact that $\sigma_{x_{0}} \Delta \sigma_{x_{1}}$ is measurable with finite measure shows that in fact the median pseudo-metric spaces $\mathcal{B}^{\mathcal{H}}{ }_{\sigma_{x_{0}}}$ and $\mathcal{B}^{\mathcal{H}}{ }_{\sigma_{x_{1}}}$ are identical: we simply denote this space by $\mathcal{B}^{\mathcal{H}}{ }_{X}$. In particular $\sigma_{x} \in \mathcal{B}^{\mathcal{H}}{ }_{X}$ for each $x \in X$.

Note that for $x, y \in X$ we have $\operatorname{pdist}_{\mu}(x, y)=\mu\left(\sigma_{x} \Delta \sigma_{y}\right)$, thus $x \mapsto \sigma_{x}$ is an isometric embedding of $X$ into $\left(\mathcal{B}^{\mathcal{H}}{ }_{X}\right.$, pdist $\left._{\mu}\right)$. Composing with the isometry $\chi^{x_{0}}: \mathcal{B}^{\mathcal{H}}{ }_{X} \rightarrow$ $\mathcal{S}^{1}(\mathcal{H}, \mu)$ we get the following well-known result stating that a wall pseudo-distance is "of type 1 ":

Lemma 3.13. Let $(X, \mathcal{W}, \mathcal{B}, \mu)$ be a space with measured walls, and let $x_{0} \in X$ denote some base point. Then the map $x \mapsto \chi_{\mathcal{W}\left(x \mid x_{0}\right)}$ defines an isometry to $L^{1}(\mathcal{W}, \mu)$. Thus if the wall pseudo-distance is a distance then $\left(X, \operatorname{dist}_{\mu}\right)$ is isometric to a subset of $L^{1}(\mathcal{W}, \mu)$, and so it is submedian.

We could probably define the median space associated to a space with measured walls $(X, \mathcal{W}, \mathcal{B}, \mu)$ to be the median hull of the isometric image of $X$ inside $L^{1}(\mathcal{W}, \mu)$ (and then perhaps take the closure in order to get a complete median space). We give here an alternative construction which is more intrinsic.

We denote by $\overline{\mathcal{M}}(X)$ the set of admissible sections, and we consider the subset $\mathcal{M}(X)=\overline{\mathcal{M}}(X) \cap \mathcal{B}^{\mathcal{H}}$. For example each admissible section $\sigma_{x}$ belongs to $\mathcal{M}(X)$, so that $X$ isometrically embeds in $\mathcal{M}(X)$. We will denote by $\iota: X \rightarrow \mathcal{M}(X)$ this isometric embedding.

Proposition 3.14. The space $\mathcal{M}(X)$ is a median subspace of $\mathcal{B}^{\mathcal{H}}$. Let $\left(X^{\prime}, \mathcal{W}^{\prime}, \mathcal{B}^{\prime}, \mu^{\prime}\right)$ be another space with measured walls. Any homomorphism of spaces with measured walls $\phi: X \rightarrow X^{\prime}$ induces an isometry $\mathcal{M}(X) \rightarrow \mathcal{M}\left(X^{\prime}\right)$. In particular the group of automorphisms of $(X, \mathcal{W}, \mathcal{B}, \mu)$ acts by isometries on $\mathcal{M}(X)$.

Proof. Considering first the picture of three points of $X$ having a median point inside $X$ (think of the case when $X$ is a tree for example), we are led to the following definition (see also Example 4.6). Given any triple $\left(\sigma_{1}, \sigma_{2}, \sigma_{3}\right) \in \mathcal{M}(X)^{3}$, let us denote by $m\left(\sigma_{1}, \sigma_{2}, \sigma_{3}\right)$ the set of half-spaces $h$ such that there exist at least two distinct indices $i, j \in\{1,2,3\}$ with $h \in \sigma_{i}, h \in \sigma_{j}$. In other words $m\left(\sigma_{1}, \sigma_{2}, \sigma_{3}\right)=\left(\sigma_{1} \cap \sigma_{2}\right) \cup\left(\sigma_{1} \cap \sigma_{3}\right) \cup\left(\sigma_{2} \cap \sigma_{3}\right)$. 
Clearly $m=m\left(\sigma_{1}, \sigma_{2}, \sigma_{3}\right)$ belongs to the set $\overline{\mathcal{M}}(X)$ of admissible sections. Fix a point $x_{0}$ in $X$ and take $\chi_{0}=\chi^{x_{0}}$ the function defined in (2). We want to show that $\chi_{0}(m)=m\left(\chi_{0}\left(\sigma_{1}\right), \chi_{0}\left(\sigma_{2}\right), \chi_{0}\left(\sigma_{3}\right)\right)$. This will prove that $m \in \mathcal{B}^{\mathcal{H}} X$ and that $m$ is a median point of $\sigma_{1}, \sigma_{2}, \sigma_{3}$.

For our set-theoretical calculation it is convenient to treat characteristic functions as maps from $\mathcal{H}$ to $\mathbb{Z} / 2 \mathbb{Z}$. We may then use the addition (mod. 2) and pointwise multiplication on these functions. We get

$$
\chi_{A \cap B}=\chi_{A} \chi_{B}, \chi_{A \triangle B}=\chi_{A}+\chi_{B}, \chi_{A \cup B}=\chi_{A}+\chi_{B}+\chi_{A} \chi_{B} .
$$

It follows easily that for any three subsets $A, B, C$ we have

$$
\chi_{(A \cap B) \cup(A \cap C) \cup(B \cap C)}=\chi_{A} \chi_{B}+\chi_{A} \chi_{C}+\chi_{B} \chi_{C} .
$$

Thus $\chi_{[(A \cap B) \cup(A \cap C) \cup(B \cap C)] \Delta D}=\chi_{A} \chi_{B}+\chi_{A} \chi_{C}+\chi_{B} \chi_{C}+\chi_{D}$. On the other hand $\chi_{((A \Delta D) \cap(B \Delta D)) \cup((A \Delta D) \cap(C \Delta D)) \cup((B \Delta D) \cap(C \Delta D))}=\left(\chi_{A}+\chi_{D}\right)\left(\chi_{B}+\chi_{D}\right)+\left(\chi_{A}+\chi_{D}\right)\left(\chi_{C}+\right.$ $\left.\chi_{D}\right)+\left(\chi_{B}+\chi_{D}\right)\left(\chi_{C}+\chi_{D}\right)=\chi_{A} \chi_{B}+\chi_{A} \chi_{C}+\chi_{B} \chi_{C}+2 \chi_{A} \chi_{D}+2 \chi_{B} \chi_{D}+2 \chi_{C} \chi_{D}+3 \chi_{D}=$ $\chi_{A} \chi_{B}+\chi_{A} \chi_{C}+\chi_{B} \chi_{C}+\chi_{D}$. We have thus checked that $[(A \cap B) \cup(A \cap C) \cup(B \cap C)] \triangle D$ coincides with $[(A \triangle D) \cap(B \triangle D)] \cup[(A \triangle D) \cap(C \triangle D)] \cup[(B \triangle D) \cap(C \triangle D)]$. Applying this to $A=\sigma_{1}, B=\sigma_{2}, C=\sigma_{3}, D=\sigma_{x_{0}}$ yields the desired result.

Consider a homomorphism of spaces with measured walls $\phi: X \rightarrow X^{\prime}$. It is easily seen that the surjective map $\phi^{*}: \mathcal{W}^{\prime} \rightarrow \mathcal{W}$ induces a surjective map $\phi^{*}: \mathcal{H}^{\prime} \rightarrow \mathcal{H}$ such that for every $B \in \mathcal{B}^{\mathcal{H}},\left(\phi^{*}\right)^{-1}(B) \in \mathcal{B}^{\mathcal{H}^{\prime}}$ and $\mu^{\prime}\left(\left(\phi^{*}\right)^{-1}(B)\right)=\mu(B)$.

Let $\sigma$ denote any admissible section. Set $\phi_{*}(\sigma)=\left(\phi^{*}\right)^{-1}(\sigma)=\left\{h^{\prime} \in \mathcal{H}^{\prime} ; \phi^{-1}\left(h^{\prime}\right) \in\right.$ $\sigma\}$. Since $\phi$ is a homomorphism, $\phi_{*}(\sigma)$ is an admissible section of $\left(X^{\prime}, \mathcal{W}^{\prime}, \mathcal{B}^{\prime}, \mu^{\prime}\right)$. Note that $\phi_{*}\left(\sigma_{x}\right)=\sigma_{\phi(x)}$ and that $\phi_{*}\left(\sigma \Delta \sigma^{\prime}\right)=\phi_{*}(\sigma) \Delta \phi_{*}\left(\sigma^{\prime}\right)$. This implies that $\phi_{*}$ defines a map from $\mathcal{M}(X)$ to $\mathcal{M}\left(X^{\prime}\right)$. Moreover pdist $\operatorname{Mi}_{\mathcal{M}\left(X^{\prime}\right)}\left(\phi_{*}(\sigma), \phi_{*}\left(\sigma^{\prime}\right)\right)=\mu^{\prime}\left(\phi_{*}(\sigma) \triangle \phi_{*}\left(\sigma^{\prime}\right)\right)=$ $\mu^{\prime}\left(\phi_{*}\left(\sigma \Delta \sigma^{\prime}\right)\right)=\mu^{\prime}\left(\left(\phi^{*}\right)^{-1}\left(\sigma \Delta \sigma^{\prime}\right)\right)=\mu\left(\sigma \Delta \sigma^{\prime}\right)=\operatorname{pdist}_{\mathcal{M}(X)}\left(\sigma, \sigma^{\prime}\right)$. Thus $\phi_{*}$ is an isometry.

The previous result justifies that we call $\mathcal{M}(X)$ the median space associated with $(X, \mathcal{W}, \mathcal{B}, \mu)$. The first part of Theorem 1.1 is proved.

Remark 3.15. The median space $\mathcal{M}(X)$ has measured walls. Indeed for each $h \in \mathcal{H}$ define $h_{\mathcal{M}}$ to be the set of $\sigma \in \mathcal{M}(X)$ such that $h \in \sigma$. The complement of $h_{\mathcal{M}}$ in $\mathcal{M}(X)$ is the set of $\sigma \in \mathcal{M}(X)$ such that $h \notin \sigma$, or equivalently by the properties of admissible sections $h^{c} \in \sigma$. In other words $\left(h_{\mathcal{M}}\right)^{c}=\left(h^{c}\right)_{\mathcal{M}}$. Thus $\left\{h_{\mathcal{M}}\right\}_{h \in \mathcal{H}}$ is a collection of half-spaces - which we will denote by $\mathcal{H}_{\mathcal{M}}$. We denote by $\mathcal{W}_{\mathcal{H}}$ the associated set of walls on $\mathcal{M}(X)$. Using the bijection $\mathcal{W} \rightarrow \mathcal{W}_{\mathcal{H}}$ induced by $h \mapsto h_{\mathcal{M}}$ we define on $\mathcal{W}_{\mathcal{H}}$ a $\sigma$-algebra $\mathcal{B}_{\mathcal{H}}$ and a measure $\mu_{\mathcal{M}}$. Note that $\iota: X \rightarrow \mathcal{M}(X)$ is a homomorphism. Note also that the distance on $\mathcal{M}(X)$ coincides with the distance induced by the measured walls structure. 
It is easy to check that the medianized space associated with $\mathcal{M}(X)$ endowed with this structure of space with measured walls is $\mathcal{M}(X)$ itself.

Remark 3.16. One cannot hope to define a median space $(\mathcal{M}(X)$, dist $)$ associated to a space with measured walls such that there exists an isometric map $\iota:\left(X\right.$, pdist $\left._{\mu}\right) \rightarrow$ $\left(\mathcal{M}(X)\right.$, dist) with the universality property that any isometric map from $\left(X\right.$, pdist $\left._{\mu}\right)$ to a median space factors through $\iota$. This was explained in Remark 2.10.

However, there is a potentially smaller medianization of a measured space with walls $X$, in which $X$ embeds isometrically, that we will call $\mathcal{M}_{f}(X)$ because it is obtained using finite subsets in $X$. We first give some naturality property of the above medianization construction in the case of a finite measured wall space.

Lemma 3.17. If $F_{1}$ and $F_{2}$ are two finite spaces with measured walls so that there is an injective homomorphism $F_{1} \hookrightarrow F_{2}$, then the following diagram commutes:

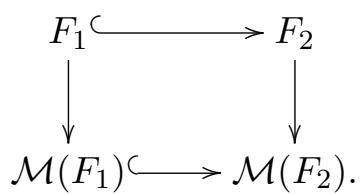

Proof. Follows directly from Proposition 3.14.

Definition 3.18. Given a space with measured walls $(X, \mathcal{W}, \mathcal{B}, \mu)$, one defines its locally finite medianization by

$$
\mathcal{M}_{f}(X)=\bigcup_{F \subseteq X \text { finite }} \mathcal{M}(F) .
$$

Remark 3.19. Notice that for a finite space with measured walls $F$, then $\mathcal{M}(F)=$ $\mathcal{M}_{f}(F)$. Moreover, since for any finite subset $F \subseteq X$, the measured wall space structure on $F$ comes from the measured wall space structure on $X$, in particular the admissible sections for $F$ will be admissible for $X$ and hence $\mathcal{M}(F) \subseteq \mathcal{M}(X)$, so that $\mathcal{M}_{f}(X) \subseteq$ $\mathcal{M}(X)$ because the latter is complete. We do not know if in general $\mathcal{M}_{f}(X)$ equals to $\mathcal{M}(X)$ or not.

3.4. Distances to the associated median space. The aim of this section is to prove that in some interesting cases, the median space associated to a space with measured wall is at finite Hausdorff distance from it.

Notation 3.20. Let $(X, \mathcal{W}, \mathcal{B}, \mu)$ be a space with measured walls. For any subset $Y \subset X$, denote by $\mathcal{W}(Y)$ the set of walls separating two points of $Y$ (we also say that these walls cut $Y$ ). The set $\mathcal{W}(Y)$ is not a priori measurable, unless $Y$ is countable for instance, in which case $\mathcal{W}(Y)=\bigcup_{y, y^{\prime} \in Y} \mathcal{W}\left(y \mid y^{\prime}\right)$.

For an arbitrary subset $Y$ we write $\bar{\mu}(\mathcal{W}(Y)) \leq K$ if for any measurable subset $\mathcal{E} \subset$ $\mathcal{W}(Y)$ we have $\mu(\mathcal{E}) \leq K$. 
Definition 3.21. We say that $(X, \mathcal{W}, \mathcal{B}, \mu)$ is $(\mu, f)$-locally finite if for any radius $R \in \mathbb{R}^{+}$ we have $\bar{\mu}(\mathcal{W}(B(x, R))) \leq f(R)<+\infty$ for every $x \in X$. When we do not want to specify $f$ we just say that $(X, \mathcal{W}, \mathcal{B}, \mu)$ is $\mu$-locally finite.

\section{Example 3.22.}

(1) The measured wall-space structure on $\mathbb{H}^{n}$ is $\mu$-locally finite. Indeed the set of walls cutting a given closed ball of $\mathbb{H}^{n}$ is compact.

(2) Discrete wall-spaces whose wall-distance is uniformly proper are $\mu$-locally finite.

(3) More generally if for each ball we have a covering $\mathcal{W}(B(x, R)) \subset \mathcal{W}\left(x \mid a_{1}\right) \cup$ $\cdots \cup \mathcal{W}\left(x \mid a_{n(R)}\right)$, with $d\left(x, a_{i}\right) \leq g(R)$, then the measured wall-space is $\mu$-locally finite.

Definition 3.23. $\left[Y_{\delta}\right.$ (pseudo-)metric spaces] Let ( $X$, pdist) be a (pseudo-)metric space. We say that $X$ is a $Y_{\delta}$ (pseudo-)metric space if given any three points $x, y, z$ there exist three points $x^{\prime}, y^{\prime}, z^{\prime}$ such that the following holds:

(1) $x^{\prime}$ is between $y$ and $z, y^{\prime}$ is between $x$ and $z, z^{\prime}$ is between $x$ and $y$;

(2) the diameter of the set $\left\{x^{\prime}, y^{\prime}, z^{\prime}\right\}$ is at most $\delta$.

Note that Gromov-hyperbolic spaces are $Y_{\delta}$, and that median spaces are $Y_{0}$.

Theorem 3.24. Let $(X, \mathcal{W}, \mathcal{B}, \mu)$ be a space with measured walls, that is $\mu$-locally finite in the sense of Definition 3.21. Assume that the wall pseudo-distance is $Y_{\delta}$. Then $\mathcal{M}(X)$ is at finite Hausdorff distance of $\iota(X)$.

As a direct consequence to this theorem we get the following.

Corollary 3.25. The inclusion $\mathbb{H}^{n} \rightarrow \mathcal{M}\left(\mathbb{H}^{n}\right)$ is a quasi-surjective isometric embedding.

We start with a technical lemma telling us that the collection of walls separating a point from a convex set differs from the collection of walls separating that point from a quasi-projection on the convex set by a set of small measure.

Lemma 3.26. Let $(X, \mathcal{W}, \mathcal{B}, \mu)$ be a space with measured walls, $(\mu, f)$-locally finite in the sense of Definition 3.21. Assume that the wall pseudo-distance pdist $_{\mu}$ is $Y_{\delta}$. Let $C$ be a convex subset of $\left(X\right.$, pdist $\left._{\mu}\right)$, let $x$ be a point outside $C$, and $p$ a quasi-projection of $x$ on $C$, that is a point of $C$ such that $\operatorname{pdist}_{\mu}(x, p)<\operatorname{pdist}_{\mu}(x, C)+\varepsilon$. Then, with the notation introduced in 3.20

$$
\bar{\mu}(\mathcal{W}(x \mid p) \backslash \mathcal{W}(x \mid C)) \leq f(2 \delta+\varepsilon) .
$$

Proof. Let $\mathcal{E}$ be a measurable subset of $\mathcal{W}$ contained in $\mathcal{W}(x \mid p) \backslash \mathcal{W}(x \mid C)$. For any wall $w=\left\{h, h^{c}\right\} \in \mathcal{E}$ assume the notation is such that $x \in h$ (so $p \in h^{c}$ ). Since the wall $w$ does not separate $x$ from $C$, the intersection $h \cap C$ is not empty. Take $q \in h \cap C$. By the $Y_{\delta}$ property there exist three points $x^{\prime}, p^{\prime}, q^{\prime}$ such that:

(1) $x^{\prime}$ is between $p$ and $q, p^{\prime}$ is between $x$ and $q, q^{\prime}$ is between $x$ and $p$;

(2) the diameter of $\left\{x^{\prime}, p^{\prime}, q^{\prime}\right\}$ with respect to pdist $_{\mu}$ is at most $\delta$. 
By convexity of $h$ and $C$, we have that $p^{\prime} \in h$ and $x^{\prime} \in C$. Now, $\operatorname{pdist}_{\mathcal{W}}\left(q^{\prime}, x^{\prime}\right) \geq$ $\operatorname{pdist}_{\mathcal{W}}\left(q^{\prime}, p\right)-\varepsilon$, because otherwise we would have that $\operatorname{pdist}_{\mu}\left(x, x^{\prime}\right)<\operatorname{pdist}_{\mu}(x, C)$. It follows that $\operatorname{pdist}_{\mathcal{W}}\left(q^{\prime}, p\right) \leq \delta+\varepsilon$ and thus $\operatorname{pdist}_{\mathcal{W}}\left(p, p^{\prime}\right) \leq 2 \delta+\varepsilon$. This shows that $w \in \mathcal{W}(B(p, 2 \delta+\varepsilon))$. Hence $\mathcal{E} \subset \mathcal{W}(B(p, 2 \delta+\varepsilon))$ and the lemma follows.

Remark 3.27. The proof of the above lemma carries over to the case where $C$ is $K$ quasiconvex only; the conclusion is then that $\bar{\mu}(\mathcal{W}(x \mid p) \backslash \mathcal{W}(x \mid C)) \leq f(2 \delta+K+\varepsilon)$. As an application we get that the convex hull of quasi-convex subsets is at finite Hausdorff distance of the convex subset (in spaces with measured wall $(X, \mathcal{W}, \mathcal{B}, \mu)$ as in the lemma above).

Proof of Theorem 3.24. Take $\tau$ an arbitrary admissible section in $\mathcal{M}(X)$, and let $x \in X$ be a point such that $\operatorname{pdist}_{\mu}\left(\tau, \sigma_{x}\right) \leq \operatorname{pdist}_{\mu}(\tau, \iota(X))+1$.

For any $y \in X$, we denote by $\mathcal{W}(y \mid \tau)$ the projection $\mathfrak{p}\left(\tau \Delta \sigma_{y}\right)$. It is composed of the walls $w=\left\{h, h^{c}\right\}$ such that $y \in h$ and $h^{c} \in \tau$. By definition $\mu(\mathcal{W}(y \mid \tau))=\operatorname{pdist}_{\mu}\left(\tau, \sigma_{y}\right)$.

We set $K=2 f(2 \delta+1)+2$ and claim that each wall in $\mathcal{W}(x \mid \tau)$ cuts the ball $B(x, K)$. The theorem follows from this claim since $(X, \mathcal{W}, \mathcal{B}, \mu)$ is $\mu$-locally finite.

We argue by contradiction and assume that there exists a wall $w=\left\{h, h^{c}\right\}$ in $\mathcal{W}(x \mid \tau)$ such that $x \in h^{c}$, and for every point $q \in h$ we have $\operatorname{pdist}_{\mu}(x, q)>K$. Let $p \in h$ be a point such that $\operatorname{pdist}_{\mu}(p, x) \leq \operatorname{pdist}_{\mu}(h, x)+1$. By Lemma 3.26 we have that

$$
\bar{\mu}(\mathcal{W}(x \mid p) \backslash \mathcal{W}(x \mid h)) \leq f(2 \delta+1) .
$$

$$
\operatorname{pdist}_{\mu}\left(\tau, \sigma_{p}\right)=\mu\left(\left(\tau \Delta \sigma_{x}\right) \Delta\left(\sigma_{p} \Delta \sigma_{x}\right)\right)=\mu(\mathcal{W}(x \mid \tau) \backslash \mathcal{W}(x \mid p))+\mu(\mathcal{W}(x \mid p) \backslash \mathcal{W}(x \mid \tau)) \text {. }
$$

Note also that $\mathcal{W}(x \mid h) \subset \mathcal{W}(x \mid \tau)$. Indeed, if the wall $w=\left\{h^{\prime}, h^{\prime c}\right\}$ is such that $x \in h^{\prime}$ and $h \subset\left(h^{\prime}\right)^{c}$, then by admissibility of $\tau$ we have that $\left(h^{\prime}\right)^{c} \in \tau$, so that $w$ separates $x$ from $\tau$.

It follows that $\mathcal{W}(x \mid p) \backslash \mathcal{W}(x \mid \tau) \subset \mathcal{W}(x \mid p) \backslash \mathcal{W}(x \mid h)$, so by Lemma 3.26 both sets have measure at most $f(2 \delta+1)$.

On the other hand $\mu(\mathcal{W}(x \mid \tau) \backslash \mathcal{W}(x \mid p))=\operatorname{pdist}_{\mu}\left(\tau, \sigma_{x}\right)-\mu(\mathcal{W}(x \mid \tau) \cap \mathcal{W}(x \mid p)) \leq$ $\operatorname{pdist}_{\mu}(\tau, \iota(X))+1-\mu(\mathcal{W}(x \mid h) \cap \mathcal{W}(x \mid p))$.

Now $\mu(\mathcal{W}(x \mid h) \cap \mathcal{W}(x \mid p))=\mu(\mathcal{W}(x \mid p))-\mu(\mathcal{W}(x \mid p) \backslash \mathcal{W}(x \mid h))$, which by Lemma 3.26 is larger than $\operatorname{pdist}_{\mu}(x, p)-f(2 \delta+1) \geq K-f(2 \delta+1)$.

We conclude that the right hand side of (3) is at most pdist $_{\mu}(\tau, \iota(X))+1+2 f(2 \delta+$ $1)-K=\operatorname{pdist}_{\mu}(\tau, \iota(X))-1$, yielding a contradiction.

Remark 3.28. (the complex hyperbolic case) As far as the complex hyperbolic space $\mathbb{H}_{\mathbb{C}}^{n}$ is concerned, less can be said: it admits a structure of space with measured walls such that the induced distance is dist ${ }^{1 / 2}$ (here dist is the hyperbolic distance), and the walls are convex with respect to the same $\operatorname{dist}^{1 / 2}$ (see Corollary 6.24). It is not known whether the same statement holds for the hyperbolic distance itself. 


\section{A Review of MEDian Algebras.}

References for this section are the book [dV93], the surveys [BH83], [Isb80], as well as the papers [Sho54a], [Sho54b] and [Rol98]. The notion of median algebra appeared as a common generalization of trees and lattices.

\subsection{Definitions, examples.}

Definition 4.1. (median algebra, first definition) A median algebra is a set $X$ endowed with a ternary operation $(a, b, c) \mapsto m(a, b, c)$ such that:

(1) $m(a, a, b)=a$;

(2) $m(a, b, c)=m(b, a, c)=m(b, c, a)$;

(3) $m(m(a, b, c), d, e)=m(a, m(b, d, e), m(c, d, e))$.

Property (3) can be replaced by $\left(3^{\prime}\right) m(a, m(a, c, d), m(b, c, d))=m(a, c, d)$.

The element $m(a, b, c)$ is the median of the points $a, b, c$. In a median algebra $(X, m)$, given any two points $a, b$ the set $I(a, b)=\{x ; x=m(a, b, x)\}$ is called the interval of endpoints $a, b$. This defines a map $I: X \times X \rightarrow \mathcal{P}(X)$. We say that a point $x \in I(a, b)$ is between $a$ and $b$.

A homomorphism of median algebras is a map $f:\left(X, m_{X}\right) \rightarrow\left(Y, m_{Y}\right)$ such that $m_{Y}(f(x), f(y), f(z))=f\left(m_{X}(x, y, z)\right)$. Equivalently, $f$ is a homomorphism if and only if it preserves the betweenness relation. If moreover $f$ is injective (bijective) then $f$ is called embedding or monomorphism (respectively isomorphism) of median algebras.

The following are straightforward properties that can be found in the literature (see for instance [Sho54a] and [Rol98, §2]).

Lemma 4.2. Let $(X, m)$ be a median algebra. For $x, y, z \in X$ we have that

(1) $I(x, x)=\{x\}$;

(2) $I(x, y) \cap I(x, z)=I(x, m(x, y, z))$;

(3) $I(x, y) \cap I(x, z) \cap I(y, z)=\{m(x, y, z)\}$;

(4) if $a \in I(x, y)$ then for any $t, I(x, t) \cap I(y, t) \subseteq I(a, t)$ (equivalently $m(x, y, t) \in$ $I(a, t))$;

(5) if $x \in I(a, b)$ and $y \in I(x, b)$ then $x \in I(a, y)$.

A sequence of points $\left(a_{1}, a_{2}, \ldots, a_{n}\right)$ is geodesic in the median algebra $(X, m)$ if $a_{i} \in$ $I\left(a_{1}, a_{i+1}\right)$ for all $i=2, \ldots, n-1$. This is equivalent, by Lemma 4.2 , point (5), to the condition that $a_{i+1} \in I\left(a_{i}, a_{n}\right)$ for all $i=1,2, \ldots, n-2$.

Lemma 4.3. If $(x, t, y)$ is a geodesic sequence, then:

(1) $I(x, t) \cup I(t, y) \subseteq I(x, y)$;

(2) $I(x, t) \cap I(t, y)=\{t\}$.

According to [Sho54a], [Sho54b] there is an alternative definition of median algebras, using intervals. 
Definition 4.4. (median algebra, second definition) A median algebra is a set $X$ endowed with a map $I: X \times X \rightarrow \mathcal{P}(X)$ such that:

(1) $I(x, x)=\{x\}$;

(2) if $y \in I(x, z)$ then $I(x, y) \subset I(x, z)$;

(3) for every $x, y, z$ in $X$ the intersection $I(x, y) \cap I(x, z) \cap I(y, z)$ has cardinality 1.

Example 4.5. Let ( $X$, dist) be a median space. Then the metric intervals $I(x, y)$ satisfy the above axioms, and thus the metric median $(x, y, z) \mapsto m(x, y, z)$ defines a structure of median algebra on $X$.

Example 4.6. Here is the set-theoretic generalization of Example 2.8(7. For any set $X$, the power set $\mathcal{P}(X)$ is a median algebra when endowed with the Boolean median operation

$$
m(A, B, C)=(A \cap B) \cup(A \cap C) \cup(B \cap C)=(A \cup B) \cap(A \cup C) \cap(B \cup C) .
$$

The median algebra $(\mathcal{P}(X), m)$ is called a Boolean median algebra. One easily sees that in this case

$$
I(A, B)=\{C ; A \cap B \subset C \subset A \cup B\} .
$$

In what follows we use the notation $\operatorname{Bm}(A, B, C)$ to designate the Boolean median defined in (4) and $B I(A, B)$ to designate the Boolean interval defined in (5).

It appears that Example 4.6 is in some sense the typical example of median algebra. More precisely, according to Corollary 4.11, any median algebra is a subalgebra of a Boolean median algebra, up to isomorphism.

\subsection{Convexity.}

Definition 4.7. A convex subset $A$ in a median algebra is a subset such that for any $a, b \in A, I(a, b) \subset A$; equivalently it is a subset such that for every $x \in X$, and $a, b$ in $A$ the element $m(a, x, b)$ is in $A$.

A subset $h$ in a median space $(X, m)$ is called a convex half-space if itself and the complementary set $h^{c}$ are convex. The pair $\left\{h, h^{c}\right\}$ is called a convex wall. We denote by $\mathcal{H}_{c}(X)$ the set of convex half-spaces in $X$ and by $\mathcal{W}_{c}(X)$ the set of convex walls in $X$. When there is no possibility of confusion we simply use the notations $\mathcal{H}_{c}$ and $\mathcal{W}_{c}$.

The above algebraic notion of convexity coincides with the metric notion of convexity introduced in Definition 2.11, in the case of the median algebra associated with a median space (see Example 4.5).

The following result shows that there are plenty of convex walls in a median algebra.

Theorem 4.8. Let $X$ be a median algebra, and let $A, B$ be two convex non-empty disjoint subsets of $X$. Then there exists a convex wall separating $A$ and $B$.

A proof of Theorem 4.8 when $A$ is a singleton can be found in [Nie78]; in its most general form it follows from [dV84, Theorem 2.5]. Other proofs can be found in [Bas01, $\S 5.2]$ and in $[\operatorname{Rol} 198, \S 2]$. 
Corollary 4.9. Given any two distinct points $x, y$ in a median space (X, dist) there exists a convex wall $w=\left\{h, h^{c}\right\}$ with $x \in h, y \in h^{c}$.

Definition 4.10. Given a median algebra $X$, one can define the map

$$
\sigma: X \rightarrow \mathcal{P}\left(\mathcal{H}_{c}\right), \sigma(x)=\sigma_{x}=\left\{h \in \mathcal{H}_{c} ; x \in h\right\} .
$$

A consequence of Theorem 4.8 is the following.

Corollary 4.11. The map $\sigma$ is an embedding of median algebras.

\section{From median spaces to spaces With measured Walls.}

The aim of this section is to prove the following.

Theorem 5.1. Let (X, dist) be a median space. Let $\mathcal{W}$ be the set of convex walls, and let $\mathcal{B}$ be the $\sigma$-algebra generated by the following subset of $\mathcal{P}(\mathcal{W})$ :

$$
\mathcal{U}=\{\mathcal{W}(x \mid y) ; x, y \text { points of } X\} .
$$

Then there exists a measure $\mu$ on $\mathcal{B}$ such that:

(1) $\mu(\mathcal{W}(x \mid y))=\operatorname{dist}(x, y)$; consequently the 4 -tuple $(X, \mathcal{W}, \mathcal{B}, \mu)$ is a space with measured walls;

(2) any isometry of ( $X$, dist) is an automorphism of the space with measured walls $(X, \mathcal{W}, \mathcal{B}, \mu)$.

Remark 5.2. According to Caratheodory's theorem, a measure $\mu$ on the $\sigma$-algebra $\mathcal{B}$ is not uniquely defined by the condition (1) in Theorem 5.1. It is uniquely defined if there exists say a sequence of points $\left(x_{n}\right)$ in $X$ such that $\mathcal{W}=\bigcup_{n, m} \mathcal{W}\left(x_{n} \mid x_{m}\right)$. This happens for instance if there exists a countable subset in $X$ whose convex hull is the entire $X$. Unicity is also guaranteed when for some topology on $\mathcal{W}$ the measure $\mu$ is borelian and $\mathcal{W}$ is locally compact second countable.

Combining Theorem 5.1 above and Lemma 3.13 we get the following:

Corollary 5.3. Any median space isometrically embeds in $L^{1}$. More precisely, given any $x_{0} \in X$, the space $X$ is isometric to $\left\{\chi_{\mathcal{W}\left(x \mid x_{0}\right)}, x \in X\right\} \subset L^{1}(\mathcal{W}, \mu)$ - or equivalently to $\left\{\mathcal{W}\left(x \mid x_{0}\right), x \in X\right\}$ endowed with $\operatorname{dist}\left(\mathcal{W}\left(x \mid x_{0}\right), \mathcal{W}\left(y \mid x_{0}\right)\right)=\mu\left(\mathcal{W}\left(x \mid x_{0}\right) \Delta \mathcal{W}\left(y \mid x_{0}\right)\right)$.

Corollary 5.4. A metric space (X, dist) is submedian in the sense of Definition 2.7 if and only if it admits a structure of space with measured walls $(X, \mathcal{W}, \mathcal{B}, \mu)$ such that dist $=$ dist $_{\mu}$. Moreover all walls in $\mathcal{W}$ may be assumed to be convex.

Proof. The direct part follows from Theorem 5.1 and Lemma 3.9.

The converse part follows from Lemma 3.13.

Theorem 5.1 together with Proposition 3.14 show that the natural dual category of median pseudo-metric spaces is the category of spaces with measured walls. Precise results on duality of categories for particular categories of median algebras and spaces with walls can be found in [Rol98] and in [Bas01]. 
Remark 5.5. According to the construction in $\S 3.2$, a space with measured walls $X$ has a natural embedding into a median space $\mathcal{M}(X)$; moreover $\mathcal{M}(X)$ has an induced structure of space with measured walls, and its metric coincides with the metric induced by the measured walls structure (Remark 3.15).

We note here that the above structure of space with measured walls on $\mathcal{M}(X)$ does not in general agree with the structure described in this section. In general the first structure does not have convex walls, as the walls on $X$ may not be convex.

The strategy of the proof of Theorem 5.1 is to use Proposition 3.5. We first show that for any pair of finite non-empty sets $F, G$ in $X, \mathcal{W}(F \mid G)$ is equal to $\mathcal{W}(a \mid b)$ for some pair of points $a, b$. In order to do this we need the following intermediate results.

Lemma 5.6. Let $(x, y, z)$ be a geodesic sequence. Then we have the following decomposition as a disjoint union:

$$
\mathcal{W}(x \mid z)=\mathcal{W}(x \mid y) \sqcup \mathcal{W}(y \mid z) .
$$

Proof. First notice that by convexity of half-spaces, the intersection $\mathcal{W}(x \mid y) \cap \mathcal{W}(y \mid z)$ is empty. Then the inclusion $\mathcal{W}(x \mid z) \subseteq \mathcal{W}(x \mid y) \cup \mathcal{W}(y \mid z)$ is clear because if a half-space $h$ contains $x$ but does not contain $z$, then either $h$ contains $y$ (in which case the wall $\left\{h, h^{c}\right\}$ separates $y$ from $z$ ) or $h^{c}$ contains $y$ (in which case the wall $\left\{h, h^{c}\right\}$ separates $x$ from $y$ ). The inclusion $\mathcal{W}(x \mid y) \cup \mathcal{W}(y \mid z) \subseteq \mathcal{W}(x \mid z)$ holds because if $h$ contains $x$ and $y \notin h$, again by convexity we cannot have $z \in h$ and hence $\left\{h, h^{c}\right\}$ separates $x$ from $z$.

As an immediate consequence we get the following.

Corollary 5.7. For any geodesic sequence $\left(x_{1}, x_{2}, \ldots, x_{n}\right)$ we have the following decomposition:

$$
\mathcal{W}\left(x_{1} \mid x_{n}\right)=\mathcal{W}\left(x_{1} \mid x_{2}\right) \sqcup \cdots \sqcup \mathcal{W}\left(x_{n-1} \mid x_{n}\right) .
$$

Corollary 5.8. If $(x, y)$ and $\left(x^{\prime}, y^{\prime}\right)$ are parallel pairs then

$$
\mathcal{W}(x \mid y)=\mathcal{W}\left(x^{\prime} \mid y^{\prime}\right)=\mathcal{W}\left(x, x^{\prime} \mid y, y^{\prime}\right) .
$$

Lemma 5.9. Given three points $x, y, z$ with median point $m$, we have $\mathcal{W}(x \mid y, z)=$ $\mathcal{W}(x \mid m)$.

Proof. According to Lemma 5.6 we have that $\mathcal{W}(x \mid y)=\mathcal{W}(x \mid m) \sqcup \mathcal{W}(m \mid y)$ and that $\mathcal{W}(x \mid z)=\mathcal{W}(x \mid m) \sqcup \mathcal{W}(m \mid z)$. It follows that

$$
\mathcal{W}(x \mid y, z)=\mathcal{W}(x \mid y) \cap \mathcal{W}(x \mid z)=\mathcal{W}(x \mid m) \sqcup(\mathcal{W}(m \mid y) \cap \mathcal{W}(m \mid z)) .
$$

But by convexity of the walls $\mathcal{W}(m \mid y) \cap \mathcal{W}(m \mid z)=\emptyset$, and we are done.

Lemma 5.10. Let $x, y, a, b$ be four points, and let $\left[a^{\prime}, x^{\prime}, b^{\prime}, y^{\prime}\right]$ be their central rectangle, as defined in Definition 2.27. Then

$$
\mathcal{W}(x \mid y) \cap \mathcal{W}(a \mid b)=\mathcal{W}\left(a^{\prime} \mid b^{\prime}\right)=\mathcal{W}\left(x^{\prime} \mid y^{\prime}\right) .
$$


Proof. According to Lemma 5.6 we may write $\mathcal{W}\left(a^{\prime} \mid b^{\prime}\right)=\mathcal{W}\left(a^{\prime} \mid x^{\prime}\right) \sqcup \mathcal{W}\left(x^{\prime} \mid b^{\prime}\right)$ and $\mathcal{W}\left(x^{\prime} \mid y^{\prime}\right)=\mathcal{W}\left(x^{\prime} \mid b^{\prime}\right) \sqcup \mathcal{W}\left(b^{\prime} \mid y^{\prime}\right)$. Corollary 5.8 implies that $\mathcal{W}\left(a^{\prime} \mid x^{\prime}\right)=\mathcal{W}\left(y^{\prime} \mid b^{\prime}\right)$, hence that $\mathcal{W}\left(a^{\prime} \mid b^{\prime}\right)=\mathcal{W}\left(x^{\prime} \mid y^{\prime}\right)$.

By Lemma 5.9 we have $\mathcal{W}\left(a \mid a^{\prime}\right)=\mathcal{W}(a \mid x, y)$. In particular $\mathcal{W}\left(a \mid a^{\prime}\right) \cap \mathcal{W}(x \mid y)=\emptyset$. And similarly $\mathcal{W}\left(b \mid b^{\prime}\right) \cap \mathcal{W}(x \mid y)=\emptyset$.

Consider now a half-space $h$ such that $a \in h, b \notin h$ and $\left\{h, h^{c}\right\} \in \mathcal{W}(x \mid y)$. Since $\mathcal{W}\left(a \mid a^{\prime}\right) \cap \mathcal{W}(x \mid y)=\emptyset$, we deduce that $a^{\prime} \in h$. Similarly we have $b^{\prime} \in h^{c}$. We have just proved that $\mathcal{W}(a \mid b) \cap \mathcal{W}(x \mid y) \subset \mathcal{W}\left(a^{\prime} \mid b^{\prime}\right)$.

On the other hand, since $\mathcal{W}\left(a^{\prime} \mid b^{\prime}\right)=\mathcal{W}\left(x^{\prime} \mid y^{\prime}\right)$ and $\left(x, x^{\prime}, y^{\prime}, y\right)$ is a geodesic, it follows that $\mathcal{W}\left(a^{\prime} \mid b^{\prime}\right) \subset \mathcal{W}(x \mid y)$.

According to Lemma $2.29,\left(a^{\prime}, b^{\prime}\right)$ is parallel to a pair $\left(a^{\prime \prime}, b^{\prime \prime}\right)$ such that $\left(a, a^{\prime \prime}, b^{\prime \prime}, b\right)$ is geodesic. This and Corollary 5.8 imply that $\mathcal{W}\left(a^{\prime} \mid b^{\prime}\right) \subset \mathcal{W}(a \mid b)$.

Proposition 5.11. Let $F$ and $G$ be two finite non-empty subsets in $X$. There exist two points $p, q \in X$ such that

$$
\mathcal{W}(F \mid G)=\mathcal{W}(p \mid q)
$$

Proof. We use an inductive argument over $n=\operatorname{card} F+\operatorname{card} G$. For $n=2$ the result is obvious, while for $n=3$ it is Lemma 5.9 .

Assume that the statement holds for $n$ and let $F, G$ be such that $\operatorname{card} F+\operatorname{card} G=$ $n+1 \geq 3$. Without loss of generality we may assume that $\operatorname{card} F \geq 2$. Then $F=$ $F_{1} \sqcup\{x\}$, and $\mathcal{W}(F \mid G)=\mathcal{W}\left(F_{1} \mid G\right) \cap \mathcal{W}(x \mid G)$. The inductive hypothesis implies that $\mathcal{W}\left(F_{1} \mid G\right)=\mathcal{W}(a \mid b)$ and $\mathcal{W}(x \mid G)=\mathcal{W}(c \mid d)$, for some points $a, b, c, d$. Hence $\mathcal{W}(F \mid G)=$ $\mathcal{W}(a \mid b) \cap \mathcal{W}(c \mid d)$. We end up by applying Lemma 5.10.

At this stage we have proven that the ring $\mathcal{R}$ defined in Proposition 3.5 coincides with the set of disjoint unions $\bigsqcup_{i=1}^{n} \mathcal{W}\left(x_{i} \mid y_{i}\right)$. It remains to show that the map $\mu$ on $\mathcal{R}$ defined by $\mu(\mathcal{W}(x \mid y))=\operatorname{dist}(x, y)$ is a premeasure on the ring $\mathcal{R}$. We first show that $\mu$ is a well-defined additive function.

Lemma 5.12. If $\mathcal{W}(x \mid y)=\mathcal{W}(a \mid b)$ then $\operatorname{dist}(x, y)=\operatorname{dist}(a, b)$.

Proof. By Lemma 5.10, if $\left[a^{\prime}, x^{\prime}, b^{\prime}, y^{\prime}\right]$ is the central rectangle of $x, y, a, b$, then $\mathcal{W}(x \mid y)=$ $\mathcal{W}(x \mid y) \cap \mathcal{W}(a \mid b)=\mathcal{W}\left(x^{\prime} \mid y^{\prime}\right)$. Since $\left(x, x^{\prime}, y^{\prime}, y\right)$ is a geodesic, this implies that $\mathcal{W}\left(x \mid x^{\prime}\right)$ and $\mathcal{W}\left(y \mid y^{\prime}\right)$ are empty, hence $x=x^{\prime}$ and $y=y^{\prime}$. In particular $\operatorname{dist}(x, y)=\operatorname{dist}\left(x^{\prime}, y^{\prime}\right)$.

Lemma 2.29 implies that $\left(a^{\prime}, b^{\prime}\right)$ is parallel to some $\left(a^{\prime \prime}, b^{\prime \prime}\right)$ such that $\left(a, a^{\prime \prime}, b^{\prime \prime}, b\right)$ is a geodesic. Then $\mathcal{W}(a \mid b)=\mathcal{W}(x \mid y) \cap \mathcal{W}(a \mid b)=\mathcal{W}\left(a^{\prime} \mid b^{\prime}\right)=\mathcal{W}\left(a^{\prime \prime} \mid b^{\prime \prime}\right)$ implies as previously that $a=a^{\prime \prime}$ and $b=b^{\prime \prime}$. Thus $\operatorname{dist}(a, b)=\operatorname{dist}\left(a^{\prime}, b^{\prime}\right)=\operatorname{dist}\left(x^{\prime}, y^{\prime}\right)=\operatorname{dist}(x, y)$.

Proposition 5.13. Assume that for two points $x, y$ the set of walls $\mathcal{W}(x \mid y)$ decomposes as $\mathcal{W}(x \mid y)=\bigsqcup_{i=1}^{n} \mathcal{W}\left(x_{i} \mid y_{i}\right)$. Then there exists a geodesic sequence $\left(a_{1}=x, a_{2}, \ldots, a_{2^{n}}=\right.$ $y)$ and a partition $\left\{1,2, \ldots, 2^{n}-1\right\}=I_{1} \sqcup I_{2} \sqcup \cdots \sqcup I_{n}$ such that:

(1) for each $j \in\{1, \ldots, n\}$ the set $I_{j}$ has $2^{j-1}$ elements and we have a decomposition of $\mathcal{W}\left(x_{j} \mid y_{j}\right)=\bigsqcup_{i \in I_{j}} \mathcal{W}\left(a_{i} \mid a_{i+1}\right)$ 
(2) for each $j \in\{1, \ldots, n\}$ we have $\operatorname{dist}\left(x_{j}, y_{j}\right)=\sum_{i \in I_{j}} \operatorname{dist}\left(a_{i}, a_{i+1}\right)$

In particular, $\operatorname{dist}(x, y)=\sum_{j} \operatorname{dist}\left(x_{j}, y_{j}\right)$ and hence the map $\mu: \mathcal{R} \rightarrow \mathbb{R}$ is uniquely defined by the condition $\mu(\mathcal{W}(x \mid y))=\operatorname{dist}(x, y)$.

To prove this Proposition we need the following auxiliary result:

Lemma 5.14. In a median space ( $X$, dist), consider two geodesic sequences with common endpoints $(x, p, q, y)$ and $\left(x, p^{\prime}, q^{\prime}, y\right)$, such that $\mathcal{W}(p \mid q) \cap \mathcal{W}\left(p^{\prime} \mid q^{\prime}\right)=\emptyset$. Let $s, t, u, v$ denote the following median points: $s=m\left(p^{\prime}, x, p\right), t=m\left(q^{\prime}, x, p\right), u=m\left(p^{\prime}, q, y\right), v=$ $m\left(q^{\prime}, q, y\right)$. Then $\operatorname{dist}\left(p^{\prime}, q^{\prime}\right)=\operatorname{dist}(s, t)+\operatorname{dist}(u, v)$.

Proof. Consider two more median points: $m=m\left(t, p^{\prime}, q^{\prime}\right), n=m\left(u, p^{\prime}, q^{\prime}\right)$ (see Figure 3 ). Then $\left[s, t, m, p^{\prime}\right]$ is a rectangle since by construction $\left(t, m, p^{\prime}\right)$ is a geodesic sequence and that $\left(q^{\prime}, m, t, s\right),\left(p^{\prime}, s, t\right)$ are geodesic follow by the usual projection-on-interval argument. And since $\left(x, p^{\prime}, q^{\prime}, y\right)$ is a geodesic sequence we see that $\left(x, s, p^{\prime}, m, q^{\prime}, y\right)$ is geodesic. Similarly one shows that $\left[u, v, q^{\prime}, n\right]$ is a rectangle.

It follows in particular that $\mathcal{W}(s \mid t)=\mathcal{W}\left(p^{\prime} \mid m\right)$ and $\mathcal{W}(u \mid v)=\mathcal{W}\left(n \mid q^{\prime}\right)$ and hence $\operatorname{dist}(s, t)=\operatorname{dist}\left(p^{\prime}, m\right)$ and $\operatorname{dist}(u, v)=\operatorname{dist}\left(n, q^{\prime}\right)$. Note that by Lemma 5.10 we have $\mathcal{W}(s \mid t)=\mathcal{W}\left(p^{\prime} \mid q^{\prime}\right) \cap \mathcal{W}(x \mid p)$ and $\mathcal{W}(u \mid v)=\mathcal{W}\left(p^{\prime} \mid q^{\prime}\right) \cap \mathcal{W}(q \mid y)$. Since $\left(x, p^{\prime}, q^{\prime}, y\right)$ is geodesic, we have $\mathcal{W}\left(p^{\prime} \mid q^{\prime}\right) \subset \mathcal{W}(x \mid y)$. Since $(x, p, q, y)$ is a geodesic sequence, $\mathcal{W}(x \mid y)=$ $\mathcal{W}(x \mid p) \sqcup \mathcal{W}(p \mid q) \sqcup \mathcal{W}(q \mid y)$. By assumption $\mathcal{W}(p \mid q) \cap \mathcal{W}\left(p^{\prime} \mid q^{\prime}\right)=\emptyset$, so we obtain $\mathcal{W}\left(p^{\prime} \mid q^{\prime}\right)=\left[\mathcal{W}(x \mid p) \cap \mathcal{W}\left(p^{\prime} \mid q^{\prime}\right)\right] \sqcup\left[\mathcal{W}(q \mid y) \cap \mathcal{W}\left(p^{\prime} \mid q^{\prime}\right)\right] . \quad$ Finally $\mathcal{W}\left(p^{\prime} \mid q^{\prime}\right)=\mathcal{W}(s \mid t) \sqcup$ $\mathcal{W}(u \mid v)=\mathcal{W}\left(p^{\prime} \mid m\right) \sqcup \mathcal{W}\left(n \mid q^{\prime}\right)$

On the other hand $\mathcal{W}\left(p^{\prime} \mid q^{\prime}\right)=\mathcal{W}\left(p^{\prime} \mid m\right) \sqcup \mathcal{W}\left(m \mid q^{\prime}\right)$, hence $\mathcal{W}\left(m \mid q^{\prime}\right)=\mathcal{W}\left(n \mid q^{\prime}\right)$, and by Lemma 5.12 it follows that $\operatorname{dist}\left(m, q^{\prime}\right)=\operatorname{dist}\left(n, q^{\prime}\right)$, which finishes the proof.

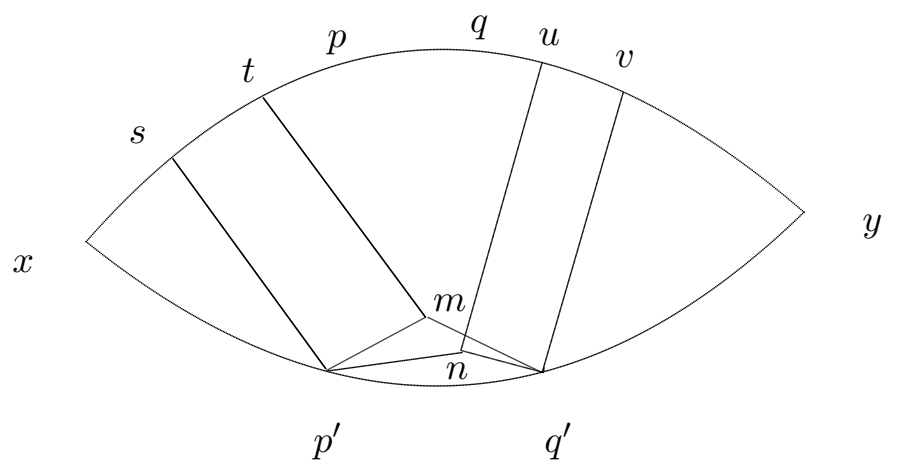

Figure 3. The construction in Lemma 5.14. 
Proof of Proposition 5.13. We argue by induction on $n$. The case $n=1$ follows by Lemma 5.12.

Now let us assume that $n>1$ and that the lemma is true for partitions of any wallinterval into $n-1$ wall-intervals. Notice first that according to Lemma 5.10, modulo replacing $x_{i}$ 's and $y_{i}$ 's by $m\left(x_{i}, x, y\right)$ and $m\left(y_{i}, x, y\right)$ respectively, we can assume that the $x_{i}$ 's and $y_{i}$ 's belong to the interval $I(x, y)$.

We denote by $p_{1}, q_{1}$ the median points $p_{1}=m\left(x, x_{1}, y_{1}\right)$ and $q_{1}=m\left(y, x_{1}, y_{1}\right)$. By Lemma $5.10\left(x, p_{1}, q_{1}, y\right)$ is a geodesic sequence, and we have $\mathcal{W}\left(x_{1} \mid y_{1}\right)=\mathcal{W}\left(p_{1} \mid q_{1}\right)$.

By Lemma 5.6 we have $\mathcal{W}(x \mid y)=\mathcal{W}\left(x \mid p_{1}\right) \sqcup \mathcal{W}\left(p_{1} \mid q_{1}\right) \sqcup \mathcal{W}\left(q_{1} \mid y\right)$. It follows that $\mathcal{W}\left(x \mid p_{1}\right) \sqcup \mathcal{W}\left(q_{1} \mid y\right)=\sqcup_{i=2}^{n} \mathcal{W}\left(x_{i} \mid y_{i}\right)$.

Consider now any pair $x_{i}, y_{i}$ with $i>1$. First replace $x_{i}, y_{i}$ by $p_{i}, q_{i}$ defined by $p_{i}=m\left(x, x_{i}, y_{i}\right), q_{i}=m\left(y, x_{i}, y_{i}\right)$ (we can do that since Lemma 5.10 tells us that we have $\mathcal{W}\left(x_{i} \mid y_{i}\right)=\mathcal{W}\left(p_{i} \mid q_{i}\right)$ and the distances coincide, that is $\left.\operatorname{dist}\left(x_{i}, y_{i}\right)=\operatorname{dist}\left(p_{i}, q_{i}\right)\right)$. Now let us project the points $p_{i}$ and $q_{i}$ onto $I\left(x, p_{1}\right)$ and $I\left(q_{1}, y\right)$. So set $s_{i}=m\left(p_{i}, x, p_{1}\right)$, $t_{i}=m\left(q_{i}, x, p_{1}\right), u_{i}=m\left(p_{i}, q_{1}, y\right)$ and $v_{i}=m\left(q_{i}, q_{1}, y\right)$.

Applying again Lemma 5.10 we get that $\mathcal{W}\left(p_{i} \mid q_{i}\right) \cap \mathcal{W}\left(x \mid p_{1}\right)=\mathcal{W}\left(s_{i} \mid t_{i}\right)$ and $\mathcal{W}\left(x_{i} \mid y_{i}\right) \cap$ $\mathcal{W}\left(q_{1} \mid y\right)=\mathcal{W}\left(u_{i} \mid v_{i}\right)$. Thus $\mathcal{W}\left(p_{i} \mid q_{i}\right)=\mathcal{W}\left(s_{i} \mid t_{i}\right) \sqcup \mathcal{W}\left(u_{i} \mid v_{i}\right)$, and we get two decompositions: $\mathcal{W}\left(x \mid p_{1}\right)=\sqcup_{i=2}^{n} \mathcal{W}\left(s_{i} \mid t_{i}\right)$ and $\mathcal{W}\left(q_{1} \mid y\right)=\sqcup_{i=2}^{n} \mathcal{W}\left(u_{i} \mid v_{i}\right)$.

If we apply the induction hypothesis to the two decompositions above we see that we are done since Lemma 5.14 ensures that $\operatorname{dist}\left(p_{i}^{\prime}, q_{i}^{\prime}\right)=\operatorname{dist}\left(s_{i}, t_{i}\right)+\operatorname{dist}\left(u_{i}, v_{i}\right)$.

The following shows that the premeasure satisfies property $\left(M_{1}^{\prime \prime}\right)$.

Proposition 5.15. Let (X, dist) be a median space, endowed with convex walls. If $\left(I_{n}\right)_{n \in \mathbb{N}}$ is a non-increasing sequence of finite disjoint unions of wall-intervals such that $\cap_{n} I_{n}=\emptyset$, then $\lim _{n \rightarrow \infty} \mu\left(I_{n}\right)=0$.

Proof. First recall that the set of convex walls (i.e. the set of convex subsets whose complement is convex as well) is a closed subset of $\{0,1\}^{X}$. In what follows we identify a half-space with its characteristic function. Then the set $\mathcal{H}(x \mid y)$ of half-spaces containing $x$ but not $y$ is a closed subset of the compact subset of $\{0,1\}^{X}$ consisting in functions $f: X \rightarrow\{0,1\}$ such that $f(x)=1, f(y)=0$. So $\mathcal{H}(x \mid y)$ is compact.

We claim that under the assumptions of the Lemma there exists $k$ such that $I_{k}=\emptyset$, which implies the conclusion.

It is enough to argue when $I_{0}=\mathcal{W}(x \mid y)$. Since $\left(I_{n}\right)_{n \in \mathbb{N}}$ is non increasing for each $n$ we have $I_{n} \subset \mathcal{W}(x \mid y)$. We then define $H_{n}$ as the set of half-spaces $h$ such that $\left\{h, h^{c}\right\} \in I_{n}$, and $x \in h$. It follows that $\left(H_{n}\right)_{n \in \mathbb{N}}$ is non increasing, and has empty intersection. By Proposition 5.13 there is a geodesic sequence $\left(x=a_{0}, a_{1}, \ldots, a_{m}=y\right)$ such that we may write $H_{n}$ as a finite (disjoint) union of some of the sets $\mathcal{H}\left(a_{j} \mid a_{j+1}\right)$. Thus each $H_{n}$ is compact, so there exists $k$ such that $H_{k}=\emptyset$, which implies that $I_{k}=\emptyset$.

We now have all the ingredients to finish the proof of Theorem 5.1. 
Proof of Theorem 5.1. That the premeasure $\mu$ is well-defined on $\mathcal{R}$ is the content of Proposition 5.13. It obviously satisfies properties $\left(M_{0}\right)$ and $\left(M_{1}^{\prime}\right)$, while $\left(M_{1}^{\prime \prime}\right)$ is proved in Proposition 5.15.

By Carathédory's theorem 3.1, $\mu^{*}$ restricted to $\mathcal{A}^{*}$ is a measure extending $\mu$, hence its restriction to $\mathcal{B}$ is also a measure extending $\mu$.

Obviously any isometry of ( $X$, dist) defines a bijective transformation on $\mathcal{W}$ preserving $\mathcal{R}$ and the premeasure $\mu$, hence the outer measure $\mu^{*}$ and $\mathcal{A}^{*}$, hence it defines an automorphism of the measured space $(\mathcal{W}, \mathcal{B}, \mu)$.

\section{KERnEls, MEDian SPACES, PROPERTy (T) AND A-T-MENABILITY}

6.1. Different types of kernels. A kernel on a set $X$ is a symmetric map $\psi: X \times X \rightarrow$ $\mathbb{R}_{+}$such that $\psi(x, x)=0$. For instance, a pseudo-metric is a kernel.

Let $f: X \rightarrow Y$ be a map and let $\phi$ be a kernel on $Y$. The pull-back of $\phi$ under $f$ is the kernel $\psi(x, y)=\phi(f(x), f(y))$. Given a class of kernels $\mathcal{C}$, a kernel $\psi$ on $X$ is of type $\mathcal{C}$ if $\psi$ is the pull-back of some kernel in the class $\mathcal{C}$.

We will be particularly interested in kernels of median type, which are obtained by pulling back a median pseudo-distance. By considering the canonical median metric quotient, we see that any kernel of median type is also the pull-back of a median distance.

Property $(\mathrm{T})$ and a-T-menability have often been described using conditionally negative definite kernels, the definition of which we now recall.

Definition 6.1. Given a set $X$, a kernel $\psi: X \times X \rightarrow \mathbb{R}_{+}$is conditionally negative definite if for every $n \in \mathbb{N}, x_{1}, \ldots, x_{n} \in X$ and $\lambda_{1}, \ldots, \lambda_{n} \in \mathbb{R}$ with $\sum_{i=1}^{n} \lambda_{i}=0$ the following holds:

$$
\sum_{i=1}^{n} \sum_{j=1}^{n} \lambda_{i} \lambda_{j} \psi\left(x_{i}, x_{j}\right) \leq 0 .
$$

Proposition 6.2 ([Sch38]). If $\psi: X \times X \rightarrow \mathbb{R}_{+}$is a conditionally negative definite kernel and $0<\alpha \leq 1$ then $\psi^{\alpha}$ is a conditionally negative definite kernel.

Proposition 6.3 ([WW75], Theorem 4.10). Let $(Y, \mathcal{B}, \mu)$ be a measured space. Let $0<p \leq 2$, and let $E=L^{p}(Y, \mu)$ endowed with the norm $\|\cdot\|_{p}$. Then $\psi: E \times E \rightarrow$ $\mathbb{R}, \psi(x, y)=\|x-y\|_{p}^{p}$ is a conditionally negative definite kernel.

In some sense, the example of conditionally negative definite kernel in Proposition 6.3 is universal, as the following result shows.

Proposition 6.4 ([Sch38]). A function $\psi: X \times X \rightarrow \mathbb{R}_{+}$is a conditionally negative definite kernel if and only if there exists a map $f: X \rightarrow H$, where $(H,\|\cdot\|)$ is a Hilbert space, such that

$$
\psi(x, y)=\|f(x)-f(y)\|^{2} .
$$

The discussion above suggests the following: 
Definition 6.5. A function $\psi: X \times X \rightarrow \mathbb{R}_{+}$is a kernel of type $p$, where $0<p \leq 2$, if there exists a map $f: X \rightarrow L^{p}(Y, \mu)$, for some measured space $(Y, \mathcal{B}, \mu)$, such that

$$
\psi(x, y)=\|f(x)-f(y)\|_{p}^{p} .
$$

Remark 6.6. Clearly, the pull-back of a conditionally negative definite kernel (or of a kernel of type $p$ ) is also conditionally negative definite (respectively, of type $p$ ).

Proposition 6.4 states that conditionally negative definite kernels are the same thing as kernels of type 2 . In order to investigate further the relationship between conditionally negative definite kernels and kernels of type $p$, we recall some results on isometric embeddings of $L^{p}$-spaces.

Theorem 6.7 (Theorems 1 and 7 in [BCK66]). Let $1 \leq p \leq q \leq 2$.

(1) The normed space $\left(L^{q}(X, \mu),\|\cdot\|_{q}\right)$ can be embedded linearly and isometrically into $\left(L^{p}\left(X^{\prime}, \mu^{\prime}\right),\|\cdot\|_{p}\right)$ for some measured space $\left(X^{\prime}, \mathcal{B}^{\prime}, \mu^{\prime}\right)$.

(2) If $L^{p}(X, \mu)$ has infinite dimension then $\left(L^{p}(X, \mu),\|\cdot\|_{p}^{\alpha}\right)$ can be embedded isometrically into $\left(L^{q}\left(X^{\prime}, \mu^{\prime}\right),\|\cdot\|_{q}\right)$ for some measured space $\left(X^{\prime}, \mathcal{B}^{\prime}, \mu^{\prime}\right)$ if and only if $0<\alpha \leq \frac{p}{q}$.

Remark 6.8. Note that according to [JR06], the space $l_{p}$ with $p>2$ does not coarsely embed into a Hilbert space.

Remark 6.9. Theorem 6.7 implies that every metric space that can be isometrically embedded in a space $L^{p}(X, \mu)$ with $p \in[1,2]$ (metric space of type $p$ in the terminology of [BCK66] and [FH74]) is a submedian space. See [FH74] for examples of such spaces.

Using these results we can now establish a more precise relation between kernels of type $p$ and conditionally negative definite.

Corollary 6.10. (1) If $\psi$ is a kernel of type $p$ for some $0<p \leq 2$ then $\psi$ is a conditionally negative definite kernel.

(2) If $\psi$ is a conditionally negative definite kernel and $1 \leq p \leq 2$ then $\psi^{\frac{p}{2}}$ is a kernel of type $p$.

Proof. Let $X$ be an arbitrary space and let $\psi: X \times X \rightarrow \mathbb{R}_{+}$.

(1) follows from Proposition 6.3 and Remark 6.6.

(2) According to Proposition 6.4 there exists a map $g: X \rightarrow L^{2}(X, \mu)$ such that $\psi(x, y)=\|g(x)-g(y)\|_{2}^{2}$. By Theorem 6.7, (1), there exists an isometric embedding $\left.F:\left(L^{2}(X, \mu),\|\cdot\|_{2}\right),\right) \rightarrow\left(L^{p}\left(X^{\prime}, \mu^{\prime}\right),\|\cdot\|_{p}\right)$. Consequently $\psi(x, y)=\|g(x)-g(y)\|_{2}^{2}=$ $\|F(g(x))-F(g(y))\|_{p}^{2}$, and $\psi^{p / 2}$ is a kernel of type $p$.

Robertson and Steger defined in [RS98] an alternate type of kernels.

Definition 6.11 (Robertson and Steger [RS98]). A measure definite kernel on a space $X$ is a map $\psi: X \times X \rightarrow \mathbb{R}_{+}$such that there exists a measured space $(\mathcal{M}, \mathcal{B}, \mu)$ and a map $S: X \rightarrow \mathcal{B}, x \mapsto S_{x}$, satisfying $\psi(x, y)=\mu\left(S_{x} \triangle S_{y}\right)$. 
In [RS98] it is asked (Question (i)) whether measure definite kernels can be given an intrinsic characterization among the conditionally negative definite kernels. It turns out that measure definite kernels are very much related to structures of space with measured walls, as well as to median spaces (see Lemma 6.12). This relationship allows us to answer this question (Proposition 6.14).

Lemma 6.12. A kernel $\psi: X \times X \rightarrow \mathbb{R}_{+}$on a space $X$ is measure definite if and only if it is of median type, in other words it is the pull-back of a median metric. Moreover when $X$ is a topological space the kernel $\psi$ is continuous if and only if the pull-back map $f$ is continuous.

Proof. Assume that $\psi$ is a measure definite kernel on $X$. Then there exists a map $S: X \rightarrow \mathcal{B}, x \mapsto S_{x}$, where $(\mathcal{M}, \mathcal{B}, \mu)$ is a measured space, and $\psi(x, y)=\mu\left(S_{x} \triangle S_{y}\right)$. It suffices then to fix some base point $x_{0}$ and to endow $\mathcal{B}_{S_{x_{0}}}$ with the structure of median pseudo-metric space described in Example 2.8(7). Then $\psi$ is the pull-back under $S$ of this median pseudo-metric.

Conversely, consider a map $f$ from $X$ to a median space ( $Y$, dist) such that $\psi\left(x, x^{\prime}\right)=$ $\operatorname{dist}\left(f(x), f\left(x^{\prime}\right)\right)$. By Theorem 5.1, there exists a set of convex walls $\mathcal{W}$ on $Y$, a $\sigma$-algebra $\mathcal{B}$ on $\mathcal{W}$ and a measure $\mu$ on $\mathcal{B}$ such that the 4 -tuple $(Y, \mathcal{W}, \mathcal{B}, \mu)$ is a space with measured walls, and moreover $\operatorname{dist}\left(y, y^{\prime}\right)=\mu\left(\mathcal{W}\left(y \mid y^{\prime}\right)\right)$.

We fix a point $x_{0}$ in $X$ and we define the map $S: X \rightarrow \mathcal{B}, S_{x}=\mathcal{W}\left(f(x) \mid f\left(x_{0}\right)\right)$. Then $\mu\left(S_{a} \Delta S_{b}\right)=\mu\left(\mathcal{W}\left(f(a) \mid f\left(x_{0}\right)\right) \Delta \mathcal{W}\left(f(b) \mid f\left(x_{0}\right)\right)\right)=\mu(\mathcal{W}(f(a) \mid f(b)))=\operatorname{dist}(f(a), f(b))=$ $\psi(a, b)$.

Obviously $f$ continuous implies $\psi$ continuous. Conversely, assume that $\psi$ is continuous. If $y \in X$ is close to $x \in X$ then $(x, y)$ is close to $(x, x)$ hence $\psi(x, y)=\operatorname{dist}(f(x), f(y))$ is close to $\psi(x, x)=0$.

The following statement is an improvement of [RS98, Proposition 1.2] and of [CMV04, Proposition 2].

Lemma 6.13. A map $\psi: X \times X \rightarrow \mathbb{R}_{+}$on a space $X$ is a measure definite kernel if and only if $X$ is endowed with a structure of space with measured walls $(X, \mathcal{W}, \mathcal{B}, \mu)$ such that $\psi\left(x, x^{\prime}\right)=\mu\left(\mathcal{W}\left(x \mid x^{\prime}\right)\right)$.

Proof. The if part follows from Lemmata 3.13 and 6.12.

Conversely, assume that $\psi$ is a measure definite kernel on $X$. By Proposition 6.12 the kernel $\psi$ is the pull-back of a median distance: $\psi(x, y)=\operatorname{dist}(f(x), f(y))$ for some map $f: X \rightarrow Y$ where ( $Y$, dist) is a median space. Consider the structure of space with measured walls on $Y$ given by Theorem 5.1. The pull-back structure of space with measured walls on $X$ has $\psi$ as wall pseudo-distance, according to Lemma 3.9.

Proposition 6.14. A kernel is measure definite if and only if it is of type 1.

Proof. Indeed if $\psi$ is measure definite then it is of median type by Lemma 6.12, and median spaces isometrically embed in some $L^{1}$-space by Corollary 5.3 . 
The converse part follows from the fact that any space $L^{1}(X, \mu)$ is a median space, and from Lemma 6.12 .

Corollary 6.15. (1) Every measure definite kernel is conditionally negative definite.

(2) If $\psi$ is a conditionally negative definite kernel then $\sqrt{\psi}$ is a measure definite kernel.

Statement (1) in Corollary 6.15 has already been proved in [RS98], where it appears as Proposition 1.1, while statement (2) has been proved in [RS98, Proposition 1.2] under the extra assumption that the set on which the kernel is defined is countable.

\subsection{Left invariant kernels, property $(\mathrm{T})$ and a-T-menability.}

Definition 6.16. A function $\Phi: G \rightarrow \mathbb{R}_{+}$defined on a group is conditionally negative definite if the function $G \times G \rightarrow \mathbb{R}_{+},(g, h) \mapsto \Phi\left(g^{-1} h\right)$, is a conditionally negative definite kernel.

Recall that a function $\Phi$ is called proper if $\lim _{g \rightarrow \infty} \Phi(g)=\infty$. Here $g \rightarrow \infty$ means that $g$ leaves any compact subset. Notice that, for any conditionally negative definite kernel $\psi: G \times G \rightarrow \mathbb{R}_{+}$which is left invariant, i.e. $\psi\left(g_{1}, g_{2}\right)=\psi\left(h g_{1}, h g_{2}\right)$, for every $h, g_{1}, g_{2}$ in $G$, the map $\Phi: G \rightarrow \mathbb{R}_{+}$defined by $\Phi(g)=\psi(1, g)$ is a conditionally negative definite function. If $\Phi$ is proper we say that the kernel $\psi$ is proper.

We also recall that a second countable space is a topological space satisfying the second axiom of countability, that is such that its topology has a countable base. A second countable space is separable (i.e. has a countable dense subset) and Lindelöf (i.e. every open cover has a countable sub-cover). The converse implications do not hold in general, but they do for metric spaces.

Characterizations of property $(\mathrm{T})$ and a-T-menability (also called the Haagerup property) using conditionally negative definite kernels are well-known and can be found in the literature, we recall here the relevant ones.

Theorem 6.17 ([Del77], [Gui77], [AW81], [dlHV89], [CCJ $\left.\left.{ }^{+} 01\right]\right)$. Let $G$ be a second countable, locally compact group.

(1) The group $G$ has property $(T)$ if and only if every continuous conditionally negative definite function on $G$ is bounded (equivalently, every continuous left invariant conditionally negative definite kernel on $G$ is bounded).

(2) The group $G$ has the Haagerup property if and only if there exists a continuous proper conditionally negative definite function on $G$ (equivalently, there exists a continuous proper left invariant conditionally negative definite kernel on $G$ ).

Theorem 6.17 and Corollary 6.10 imply the following.

Corollary 6.18. Let $G$ be a second countable, locally compact group.

(1) If the group $G$ has property $(T)$ then for every $p \in(0,2]$, every continuous left invariant kernel of type $p$ on $G$ is bounded. 
(2) The group $G$ has the Haagerup property if for some $p \in(0,2]$, there exists a continuous proper left invariant kernel of type $p$ on $G$.

Remark 6.19. For $p \in[1,2]$ the converse statements in Corollary 6.18 immediately follow from Corollary 6.10, (2).

Corollary 6.18 can be reformulated in terms of actions of the group on subsets of $L^{p}$-spaces, as follows.

Corollary 6.20. Let $G$ be a second countable, locally compact group.

(1) If the group $G$ has property $(T)$ then for every $p \in(0,2]$, any continuous action by isometries of $G$ on any subset of any space $L^{p}(X, \mu)$ has bounded orbits.

(2) The group $G$ has the Haagerup property if for some $p \in(0,2]$, there exists a continuous proper action by isometries of $G$ on a subset of some $L^{p}(X, \mu)$.

Proof. Both (1) and (2) follow from the fact that if $S$ is a subset of some $L^{p}(X, \mu)$ and there exists an action of $G$ on $S$ by isometries, $G \times S \rightarrow S,(g, s) \mapsto g \cdot s$, then for any $s \in S$ the map $\psi(g, h)=\|g \cdot s-h \cdot s\|_{p}^{p}$ is a continuous left invariant kernel of type $p$ on $G$.

The converse statements in Corollary 6.20 follow from the fact that given $\psi$ a continuous proper left invariant kernel of type $p$ on $G$, that is a map $\psi: G \times G \rightarrow \mathbb{R}_{+}$defined by $\psi(g, h)=\|f(g)-f(h)\|_{p}^{p}$, where $f: G \rightarrow L^{p}(X, \mu)$ is continuous, one can define a continuous action of $G$ on $f(G)$ by isometries by $g \cdot f(h)=f(g h)$.

For $p=1$ in Corollary 6.20, the sufficient condition to have property (T) can be weakened: it suffices to look at actions of $G$ on median subspaces of $L^{1}(X, \mu)$. Also, Haagerup property implies more for $p=1$ : the existence of a continuous proper action by isometries of $G$ on a median subspace of some $L^{1}(X, \mu)$. Both statements are consequences of the following result.

Theorem 6.21. Let $G$ be a separable topological group. $A$ map $\psi: G \times G \rightarrow \mathbb{R}_{+}$ is a continuous left invariant kernel of type 1 if and only if there exists a continuous action by isometries of $G$ on a median space (X, dist), and a point $x \in X$ such that $\psi\left(g, g^{\prime}\right)=\operatorname{dist}\left(g \cdot x, g^{\prime} \cdot x\right)$.

We first need to establish equivariant versions of Lemmata 6.12 and 6.13 when $X$ is a group $G$. In the particular case when the group is countable, Lemma 6.13 has the following equivariant version.

Proposition 6.22 ([RS98]). Let $\Gamma$ be a countable group. A map $\psi: \Gamma \times \Gamma \rightarrow \mathbb{R}_{+}$is a left invariant measure definite kernel if and only if $\Gamma$ is endowed with a left invariant structure of space with measured walls $(\Gamma, \mathcal{W}, \mathcal{B}, \mu)$ such that $\psi\left(g, g^{\prime}\right)=\mu\left(\mathcal{W}\left(g \mid g^{\prime}\right)\right)$.

This immediately implies the following equivariant version of Lemma 6.12. 
Lemma 6.23. Let $\Gamma$ be a countable group. A map $\psi: \Gamma \times \Gamma \rightarrow \mathbb{R}_{+}$is a left invariant measure definite kernel if and only if there exists an action by isometries of $\Gamma$ on a median space $(X$, dist $)$, and a point $x \in X$ such that $\psi\left(g, g^{\prime}\right)=\operatorname{dist}\left(g \cdot x, g^{\prime} \cdot x\right)$.

Proof of Theorem 6.21. The if part follows since median spaces isometrically embed in $L^{1}$-spaces by Corollary 5.3 .

We now turn to the converse. Let $\Gamma$ be a countable dense subgroup in $G$. Restrict $\psi$ to $\Gamma$ and apply Propositions 6.22 and 3.14: there exists an action by isometries of $\Gamma$ on a median space $\left(X\right.$, dist) and $x \in X$ such that $\psi\left(\gamma, \gamma^{\prime}\right)=\operatorname{dist}\left(\gamma \cdot x, \gamma^{\prime} \cdot x\right)$. The metric completion of $(X$, dist) is still median by Proposition 2.21 , and any isometry of $X$ extends uniquely to an isometry of the completion. We get an action of $\Gamma$ on a complete median space that still induces the kernel $\psi$. Thus we may - and will - assume that the median space $(X$, dist) is already complete.

The map $f: \Gamma \rightarrow X$ sending $\gamma$ to $\gamma \cdot x$ is continuous since $\psi$ is continuous (we endow $\Gamma \subset G$ with the induced topology). Since $X$ is complete it follows that $f$ extends to a map $G \rightarrow X$ still verifying $\psi\left(g, g^{\prime}\right)=\operatorname{dist}\left(g \cdot x, g^{\prime} \cdot x\right)$ (for all $g, g^{\prime}$ in $G$ ). As usual the left invariance of $\psi$ implies that $g \cdot f(h)=f(g h)$ defines an action of $G$ by isometries on $f(G)$. And the continuity of $\psi$ implies that the action is continuous.

To end the argument it suffices to prove that the above action of $G$ on $f(G)$ extends to an action by isometries on the median hull of $f(G)$ in $X$, which we denote by $M$. Set $M_{0}=f(G)$ and then define inductively $M_{i+1}=\left\{m(x, y, z) \mid(x, y, z) \in M_{i}\right\}$. Clearly $M$ is the ascending union of the $M_{i}$ 's. There is at most one isometric embedding $\bar{g}: M \rightarrow X$ extending $g: M_{0} \rightarrow M_{0}$, and such a map must satisfy $\bar{g}(M) \subset M$. Indeed since isometries commute with the median map, $\bar{g}$ is completely determined on $M_{1}$ and $\bar{g}\left(M_{1}\right) \subset M_{1}$, then $\bar{g}$ is completely determined on $M_{2}$ and $\bar{g}\left(M_{2}\right) \subset M_{2}$, and so on. We now prove the existence of such an isometric extension.

Choose a sequence $\gamma_{n}$ of elements of $\Gamma$ such that $\psi\left(\gamma_{n}, g\right) \rightarrow 0$, so that $\gamma_{n}$ converges pointwise to $g$ on $M_{0}$. We claim that for any $m \in M$ the sequence $\gamma_{n}(m)$ converges. Indeed this is certainly true for $m \in M_{0}$. Assume we know that $\gamma_{n}(p)$ converges for every $p \in M_{i}$, and let $m$ denote an element of $M_{i+1}$. Write $m=m(x, y, z)$ with $(x, y, z) \in\left(M_{i}\right)^{3}$. Since $\gamma_{n}$ acts by isometry on the whole space $X$ we have $\gamma_{n}(m)=$ $m\left(\gamma_{n}(x), \gamma_{n}(y), \gamma_{n}(z)\right)$. By induction the three sequences $\left(\gamma_{n}(x)\right)_{n \geq 0},\left(\gamma_{n}(y)\right)_{n \geq 0}\left(\gamma_{n}(z)\right)_{n \geq 0}$ are convergent. By the continuity of the median map (Corollary 2.15) it follows that $\left(\gamma_{n}(m)\right)_{n \geq 0}$ converges. Denote $\bar{g}: M \rightarrow X$ the pointwise limit of $\gamma_{n}$ on $M$. It immediately follows that $\bar{g}$ is an isometric embedding which extends $g: M_{0} \rightarrow M_{0}$. By the remarks above concerning uniqueness we have in fact $\bar{g}(M) \subset M$.

Using the uniqueness of the extension it is now straightforward to check that the maps $\bar{g}$ are isometries of $M$ (with inverse $\overline{g^{-1}}$ ), and finally that $g \mapsto \bar{g}$ defines an action of $G$ by isometries on $M$ that extends the action of $G$ on $f(G)$, and thus still induces the kernel $\psi$.

Theorem 6.21 allows to obtain some results concerning a structure of space with measured walls on the complex hyperbolic space. 
Corollary 6.24 (walls in the complex hyperbolic case). The complex hyperbolic space $\mathbb{H}_{\mathbb{C}}^{n}$ admits a structure of space with measured walls such that:

(1) the induced metric is dist $^{1 / 2}$, where dist is the hyperbolic distance;

(2) the walls are all the convex walls with respect to dist ${ }^{1 / 2}$;

(3) $S U(n, 1)$ acts by isomorphisms on this structure.

Proof. According to [FH74] the complex hyperbolic space $\mathbb{H}_{\mathbb{C}}^{n}$ endowed with the metric dist $^{1 / 2}$, where dist is the hyperbolic distance, can be embedded into a Hilbert space. It follows, by Theorem 6.7, (1), that $\psi: \mathbb{H}_{\mathbb{C}}^{n} \times \mathbb{H}_{\mathbb{C}}^{n} \rightarrow \mathbb{R}_{+}, \psi(x, y)=\operatorname{dist}^{1 / 2}(x, y)$ is a kernel of type 1 , in the terminology of Definition 7 . Obviously $\psi$ is left-invariant with respect to the action of $G=S U(n, 1)$.

Via the identification of $\mathbb{H}_{\mathbb{C}}^{n}$ with $G / K$, where $K=S U(n)$, the kernel $\psi$ induces a left invariant pull-back kernel $\psi_{G}: G \times G \rightarrow \mathbb{R}_{+}$. Theorem 6.21 implies that $G$ acts by isometries on a median space $\left(X, \operatorname{dist}_{X}\right)$ such that $\psi_{G}\left(g, g^{\prime}\right)=\operatorname{dist}_{X}\left(g x, g^{\prime} x\right)$ for some $x \in X$. It follows easily that the map $g \mapsto g x$ factorizes to a $G$-equivariant isometric embedding $g K \mapsto g x$ of $\left(\mathbb{H}_{\mathbb{C}}^{n}\right.$, dist $\left.{ }^{1 / 2}\right)$ into $\left(X\right.$, dist $\left._{X}\right)$. All the statements in the Corollary then follow from Lemma 3.9 and from Theorem 4.8 .

We now prove the results stated in the introduction.

Proof of Theorem 1.4. By Corollary 6.18 and Remark 6.19, property (T) and a-T-menability for a group $G$ are characterized by properties of continuous left invariant kernels of type 1 . But by Theorem 6.21 such kernels are induced by actions of $G$ on median spaces. The theorem follows since bounded kernels correspond to actions with bounded orbits, and proper kernels correspond to proper actions.

Proof of Theorem 1.5. If a group acts on a median space by isometries then the group acts by isomorphisms on the structure of measured walls associated to it, by Theorem 5.1. This and Theorem 1.4 give the direct implication in (2) and the converse implication in (1).

On the other hand, if a group acts by isomorphisms on a space with measured walls $X$ then by Proposition 3.14 it acts by isometries on the median space $\mathcal{M}(X)$ associated with $X$. Moreover the action on $\mathcal{M}(X)$ is proper or with bounded orbits if and only if the action on $X$ has these properties with respect to the wall pseudo-metric. Together with Theorem 1.4 this yields the direct implication in (1) and the converse implication in (2).

Proof of Corollary 1.6. An action of the group $G$ on a space with measured walls $(X, \mathcal{W}, \mathcal{B}, \mu)$ induces by Lemma 3.11 an action by affine isometries on $L^{p}\left(\mathcal{H}, \mu_{\mathcal{H}}\right)$ for any $p \geq 1$, defined by $g \cdot f=\pi_{p}(g)(f)+\chi_{\sigma_{g x}}-\chi_{\sigma_{x}}$, where $x$ is an arbitrary point in $X$.

The hypothesis in case (1) implies that the orbit of the constant function zero is composed of the functions $\chi_{\sigma_{g x}}-\chi_{\sigma_{x}}, g \in G$, and the fact that it is bounded implies that the orbit of $x$ is bounded. 
(2) If $G$ is a-T-menable then $\operatorname{pdist}_{\mu}(x, g x) \rightarrow \infty$ when $g \rightarrow \infty$, hence the action of $G$ on $L^{p}\left(\mathcal{H}, \mu_{\mathcal{H}}\right)$ is proper.

One may ask whether Theorem 1.4 cannot be improved to a generalization of the Bass-Serre theory for actions of groups on median spaces. It is indeed the case for median graphs, which can be identified to 1-skeleta of CAT(0) cube complexes ([Ger97], [Ger98], [Che00]). V. Gerasimov showed that if $G$ is a finitely generated group acting on a CAT(0) cube complex without a fixed point then there exists a stabilizer $H$ of some convex wall such that $e(G, H)>1$. Here $e(G, H)$ stands for the number of ends of the group $G$ with respect to the subgroup $H$, in the sense of [Hou74]. Conversely, V. Gerasimov showed that any group $G$ that has a subgroup $H$ with $e(G, H)>1$ acts on a $\operatorname{CAT}(0)$ cube complex without a fixed point, so that $H$ is a finite index subgroup in the stabilizer of a convex wall ([Ger97], [Ger98]). These improve previous results of M. Sageev [Sag95] (see also [NSSS05]).

Question 6.25. Is there a generalization of the Rips theory for actions of groups on median spaces?

Note that such a theory combined with Proposition 7.14 would allow to obtain many applications similar to the ones of the classical Rips theory.

We may also generalize the dynamical characterization of property $(\mathrm{T})$ obtained in [RS98], and add a dynamical characterization of a-T-menability, as follows.

Theorem 6.26. Let $G$ be a locally compact second countable group.

(1) The group $G$ has property $(T)$ if and only if for every measure-preserving action of $G$ on a measure space $(X, \mathcal{B}, \mu)$ and every set $S \subset X$ such that for all $g \in G$, $S \triangle g S$ is in $\mathcal{B}$ and $\mu(S \triangle g S)<\infty$, the supremum $\sup _{g \in G} \mu(S \triangle g S)$ is finite.

(2) The group $G$ is a-T-menable if and only if there exists a measure-preserving action of $G$ on a measure space $(X, \mathcal{B}, \mu)$ and there exists a set $S \subset \mathcal{B}$ such that for all $g \in G, S \triangle g S$ is in $\mathcal{B}$ and $\mu(S \triangle g S)<\infty$, but $\mu(S \triangle g S) \rightarrow \infty$ when $g \rightarrow \infty$.

Proof of Theorem 6.26. The only if part of (1) and the if part of (2) immediately follow from Corollary 6.18 and Proposition 6.14.

The if part of (1) and the only if part of (2) follow from Theorem 1.4 and from Corollary 5.3.

\section{QUASIFICATIONS AND FURTHER EXAMPLES.}

Definition 7.1. Let ( $X$, dist) be a metric space, and $\delta$ a non-negative real number.

A finite sequence of points $\left(a_{1}, a_{2}, \ldots, a_{n}\right)$ is a $\delta$-discrete geodesic sequence if $\operatorname{dist}\left(a_{i}, a_{i+1}\right) \leq$ $\delta$ for every $i=1,2, \ldots, n-1$, moreover $\left(a_{1}, a_{2}, \ldots, a_{n}\right)$ is a $\delta$-geodesic sequence in the sense of Definition 2.16, that is

$$
\operatorname{dist}\left(a_{1}, a_{2}\right)+\operatorname{dist}\left(a_{2}, a_{3}\right)+\cdots+\operatorname{dist}\left(a_{n-1}, a_{n}\right) \leq \operatorname{dist}\left(a_{1}, a_{n}\right)+\delta .
$$


We say that a metric space $(X$, dist) is $\delta$-discretely geodesic if there exists a constant $\delta>0$ such that for every two points $x, y$ there exists a $\delta$-discrete geodesic sequence of endpoints $x$ and $y$.

Definition 7.2. An $L_{\delta}$-space is a space such that for any three points $x, y, z$ the set $M_{\delta}(x, y, z)$ of $\delta$-median points, defined in Definition 2.19 is non-empty.

A metric space $\left(X\right.$, dist) is $\delta$-quasi-median if it is $L_{\delta}$ and moreover the diameter of all $M_{\delta}(x, y, z)$ is bounded uniformly in $x, y, z$.

A metric space $(X$, dist) is strongly quasi-median if it is $\delta$-quasi-median for some $\delta \geq 0$ and moreover for every $D \geq \delta$ the diameter of all $M_{D}(x, y, z)$ is uniformly bounded.

Let $\phi: \mathbb{R}_{+} \rightarrow \mathbb{R}_{+}$be an increasing function such that for every $D \geq \delta$ the diameter of all $M_{D}(x, y, z)$ is at most $\phi(D)$. We say that $(X$, dist) is a $\phi$-strongly quasi-median space.

Remark 7.3. It follows immediately from Lemma 2.20 that any median space is (linearly) quasi-median (it is $\phi$-median with $\phi(\delta)=6 \delta$ ).

Example 7.4. Any $\delta$-hyperbolic metric space is strongly quasi-median with $\phi(t)=\alpha t+\beta$ for some non-negative $\alpha, \beta$.

Example 7.5. The group $\mathbb{Z}^{2}$ equipped with the Euclidean distance is not quasi-median. In particular quasi-median is not a quasi-isometry invariant.

In fact a given finitely generated group $\Gamma$ may have two finite generating sets $S_{1}, S_{2}$ such that $\left(\Gamma\right.$, dist $\left._{S_{1}}\right)$ is median but $\left(\Gamma, \operatorname{dist}_{S_{2}}\right)$ is not quasi-median: take $\Gamma=\mathbb{Z}^{2}, S_{1}=$ $\{(1,0),(0,1)\}, S_{2}=\{(1,0),(0,1),(1,-1)\}$.

Remark 7.6 ((quasi-)median groups). Since being (quasi-)median is not a quasi-isometry invariant, one cannot say that a finitely generated group is (quasi-)median without specifying the metric with respect to which this property holds. This metric may be a word metric, or a metric induced by a properly discontinuous cocompact free action on a metric space.

Thus, when stating that a finitely generated group is (quasi-)median we shall always mean that the group has these properties with respect to a metric that is discretely geodesic and quasi-isometric to a word metric on the group.

Question 7.7. Are there criteria allowing to recognize groups on which there exists a discretely geodesic metric quasi-isometric to a word metric turning the group into a (quasi-)median metric space ?

Remark 7.8. A discrete group may be strongly quasi-median without being median. For instance an infinite hyperbolic group with property $(\mathrm{T})$ is strongly quasi-median. On the other hand if it would be median then this would mean that it acts on a median space with unbounded orbits (see Corollary 6.20). 
Definition 7.9. A subset $Y \subset X$ is $(\delta, K)$-convex for some $\delta \geq 0$ if given any two points $x, y$ in $Y$ the set $I_{\delta}(x, y)$ is contained in $\overline{\mathcal{N}}_{K}(Y)$ for a constant $K \geq 0$ uniform in $x$ and $y$.

Remark 7.10. A $(\delta, K)$-convex subset of an $L_{\delta}$ ( $\phi$-strongly quasi-median) space is itself an $L_{\delta+2 K}$ ( $\phi$-strongly quasi-median) space.

Given two functions $f, g:[a,+\infty) \rightarrow \mathbb{R}$ we write $f \ll g$ if for every $x \in[a,+\infty)$, $f(x) \leq g(b x+c)+d$, where $b, c, d$ are real numbers $b>0, c \geq 0, d \geq 0$. We write $f \asymp g$ if $f \ll g$ and $f \gg g$.

Proposition 7.11 (relatively hyperbolic groups). Let $G$ be a finitely generated group hyperbolic relative to a finite family of finitely generated subgroups $H_{1}, \ldots, H_{n}$. Let dist be a metric on $G$ discretely geodesic and quasi-isometric to a word metric. The following hold:

(1) $\left(G\right.$, dist) is an $L_{\delta}$-space for some $\delta>0$ if and only if $\left(H_{i}\right.$, dist) is $L_{\delta^{\prime}}$ for some $\delta^{\prime}>0$ and any $i \in\{1, \ldots, n\}$.

(2) $\left(G\right.$, dist) is $\phi$-strongly quasi-median for some increasing function $\phi: \mathbb{R}_{+} \rightarrow \mathbb{R}_{+}$ if and only if $\left(H_{i}\right.$, dist) is $\phi^{\prime}$-strongly quasi-median for some increasing function $\phi^{\prime}: \mathbb{R}_{+} \rightarrow \mathbb{R}_{+}$and any $i \in\{1, \ldots, n\}$. Moreover, $\phi^{\prime} \asymp \phi$.

Proof. In [DS05b] and in [Dru06] are given several definitions of relative hyperbolicity using only a list of metric properties. Since this list is invariant with respect to quasiisometry, it holds, as well as all the other metric properties deduced from it, for any geodesic metric on $G$ quasi-isometric to a word metric, in particular for dist. It follows by [DS05b, Lemma 4.3] that for any $\delta$ there exists $K_{\delta}$ such that all $H_{i}, i \in\{1,2, \ldots, n\}$, are $\left(\delta, K_{\delta}\right)$-convex. This and Remark 7.10 imply the direct part of the implications in (1) and (2).

The converse implication of (1) is a consequence of property $\left(^{*}\right)$ of relatively hyperbolic groups, introduced in [DS05a] and further studied in [Dru06]. According to this property the following holds:

Lemma 7.12 ([DS05a], Proposition 2.9). For every three points $x, y, z$ in $G$ there exist a left coset $g H_{i}, i \in\{1,2, \ldots, n\}$, and three points $x^{\prime}, y^{\prime}, z^{\prime}$ in $g H_{i}$ such that $\left(x, x^{\prime}, y^{\prime}, y\right)$, $\left(x, x^{\prime}, z^{\prime}, z\right)$ and $\left(y, y^{\prime}, z^{\prime}, z\right)$ are $\delta$-geodesic sequences.

We now prove the converse implication of (2). Let $m, m^{\prime}$ be two points in $M(x, y, z)$, where $x, y, z$ are arbitrary pairwise distinct points in $G$. In what follows we denote by $[a, b]$ a discrete geodesic sequence in $(G$, dist) joining two points $a, b \in G$. We denote by $[a, b] \cup[b, c]$ the discrete geodesic sequence obtained by concatenating the two geodesic sequences $[a, b]$ and $[b, c]$.

The geodesic sequences $[x, m] \cup[m, y]$ and $\left[x, m^{\prime}\right] \cup\left[m^{\prime}, y\right]$ are quasi-geodesics. According to [DS05b, Lemma 4.25] there exists a constant $\kappa=\kappa\left(G\right.$, dist) such that $m^{\prime}$ is either at distance $\kappa$ from $[x, m] \cup[m, y]$ or at distance $\kappa$ from a left coset $g H$ with $H \in\left\{H_{1}, \ldots, H_{n}\right\}$ and $\mathcal{N}_{\kappa}(g H)$ intersecting $[x, m] \cup[m, y]$. 
CAse 1. Assume that $m^{\prime}$ is at distance $\kappa$ from $[x, m]$. Then $m^{\prime} \in I_{2 \kappa}(x, m) \subset$ $I_{\delta+2 \kappa}(x, y) \cap I_{\delta+2 \kappa}(x, z)$. It follows that $m \in I_{\delta+2 \kappa}\left(m^{\prime}, y\right) \cap I_{\delta+2 \kappa}\left(m^{\prime}, z\right)$. We may then write $\operatorname{dist}(y, z)+\delta \geq \operatorname{dist}\left(y, m^{\prime}\right)+\operatorname{dist}\left(m^{\prime}, z\right) \geq 2 \operatorname{dist}\left(m, m^{\prime}\right)+\operatorname{dist}(m, y)+\operatorname{dist}(m, z)-$ $2 \delta-4 \kappa \geq 2 \operatorname{dist}\left(m, m^{\prime}\right)+\operatorname{dist}(y, z)-3 \delta-4 \kappa$. It follows that $\operatorname{dist}\left(m, m^{\prime}\right) \leq 2 \delta+2 \kappa$.

CASE 2.A We now assume that $m^{\prime}$ is at distance $\kappa$ from a left coset $g H$ such that $\mathcal{N}_{\kappa}(g H)$ intersects $[x, m] \cup[m, y]$. The entrance points $u$ and respectively $\bar{u}$ into $\overline{\mathcal{N}}_{\kappa}(g H)$ of the quasi-geodesics $[x, m] \cup[m, y]$ and respectively $\left[x, m^{\prime}\right] \cup\left[m^{\prime}, y\right]$ are at distance at most $\lambda$ for some $\lambda=\lambda(G$, dist), by [DS05b, Corollary 8.14]. Likewise for the two respective exit points $v$ and $\bar{v}$ from $\overline{\mathcal{N}}_{\kappa}(g H)$ of $[x, m] \cup[m, y]$ and respectively $\left[x, m^{\prime}\right] \cup\left[m^{\prime}, y\right]$. Assume that both $u$ and $v$ are on $[x, m]$. Then $m^{\prime} \in I_{4 \lambda}(u, v) \subset I_{4 \lambda}(x, m)$ and an argument as in Case 1 implies that $\operatorname{dist}\left(m, m^{\prime}\right) \leq 2 \delta+4 \lambda$.

CASE 2.B Thus we may assume that $u \in[x, m]$ and $v \in[m, y]$. By the quasiconvexity of left cosets of peripheral subgroups in a relatively hyperbolic group [DS05b, Lemma 4.3] it follows that $[u, m] \cup[m, v] \subset \overline{\mathcal{N}}_{\tau \kappa}(g H)$ for some $\tau=\tau(G$, dist).

With similar arguments we may assume that there exist left cosets $g^{\prime} H^{\prime}$ and $g^{\prime \prime} H^{\prime \prime}$ such that $m^{\prime} \in \mathcal{N}_{\kappa}\left(g^{\prime} H^{\prime}\right) \cap \mathcal{N}_{\kappa}\left(g^{\prime \prime} H^{\prime \prime}\right)$ and $[x, m] \cup[m, z]$ enters $\overline{\mathcal{N}}_{\kappa}\left(g^{\prime} H^{\prime}\right)$ in a point $u^{\prime} \in[x, m]$, exits it in a point $w^{\prime} \in[m, z]$ and between $u^{\prime}$ and $w^{\prime}$ is contained in $\overline{\mathcal{N}}_{\tau \kappa}\left(g^{\prime} H^{\prime}\right)$; likewise $[y, m] \cup[m, z]$ enters $\overline{\mathcal{N}}_{\kappa}\left(g^{\prime \prime} H^{\prime \prime}\right)$ in a point $v^{\prime \prime} \in[y, m]$, exits it in a point $w^{\prime \prime} \in[m, z]$, and between $v^{\prime \prime}$ and $w^{\prime \prime}$ it is contained in $\overline{\mathcal{N}}_{\tau \kappa}\left(g^{\prime \prime} H^{\prime \prime}\right)$.

According to [DS05b, Theorem 4.1], for two distinct left cosets $g H \neq g^{\prime} H^{\prime}$, the intersection $\overline{\mathcal{N}}_{\tau \kappa}(g H) \cap \overline{\mathcal{N}}_{\tau \kappa}\left(g^{\prime} H^{\prime}\right)$ has diameter at most $D_{\tau \kappa}$. If $\operatorname{dist}(u, m) \leq D_{\tau \kappa}$ then $m$ is at distance at most $D_{\tau \kappa}+\kappa$ from $\left[x, m^{\prime}\right]$ and an argument as in Case 1 allows to conclude that $\operatorname{dist}\left(m, m^{\prime}\right) \leq 2 \delta+2\left(D_{\tau \kappa}+\kappa\right)$.

We may therefore assume that $\operatorname{dist}(u, m)>D_{\tau \kappa}$ and also $\operatorname{dist}\left(u^{\prime}, m\right)>D_{\tau \kappa}$. It follows that $\overline{\mathcal{N}}_{\tau \kappa}(g H) \cap \overline{\mathcal{N}}_{\tau \kappa}\left(g^{\prime} H^{\prime}\right)$ has diameter larger than $D_{\tau \kappa}$, hence $g H=g^{\prime} H^{\prime}$. With a similar argument we obtain that $g H=g^{\prime \prime} H^{\prime \prime}$.

Thus there exist $u \in[x, m], v \in[y, m]$ and $w \in[z, m]$ in $\overline{\mathcal{N}}_{\kappa}(g H)$, and $\bar{u} \in\left[x, m^{\prime}\right]$, $\bar{v} \in\left[y, m^{\prime}\right]$ and $\bar{w} \in\left[z, m^{\prime}\right]$ in $\overline{\mathcal{N}}_{\kappa}(g H)$ such that $\operatorname{dist}(u, \bar{u})$, $\operatorname{dist}(v, \bar{v})$ and $\operatorname{dist}(w, \bar{w})$ are at most $\lambda$. This and the hypothesis that $(H$, dist $)$ is $\phi^{\prime}$-strongly quasi-median implies that $G$ is $\phi$-strongly quasi-median with $\phi \ll \phi^{\prime}$.

In particular this allows to state that in a relatively hyperbolic group, if the peripheral subgroups are $L_{\delta}$ or strongly quasi-median with respect to a good metric then the whole group has a good metric with respect to which it has the same property.

Corollary 7.13. Let $G$ be a finitely generated group hyperbolic relative to a finite family of finitely generated subgroups $H_{1}, \ldots, H_{n}$. Assume that for every $i \in\{1, \ldots, n\}$ the subgroup $H_{i}$ admits a metric dist ${ }_{i}$ discretely geodesic and quasi-isometric to a word metric with respect to which $H_{i}$ are $L_{\delta}$ ( $\phi$-strongly quasi-median).

Then the group $G$ admits a metric discretely geodesic and quasi-isometric to a word metric with respect to which $G$ is $L_{\delta}$ (respectively $\phi^{\prime}$-strongly quasi-median, with $\left.\phi^{\prime} \ll \phi\right)$. 
Proof. Let $S_{i}$ be a finite set of generators for $H_{i}$ and let $S$ be a finite set of generators for $G$ containing all $S_{i}, i=1,2, \ldots, n$. In the Cayley graph of $G$ with respect to $S$ consider for every pair of distinct left cosets $g H_{i}, g^{\prime} H_{j}$ a geodesic minimizing the distance.

Let $\delta_{i}$ be such that $\left(H_{i}\right.$, dist $\left._{i}\right)$ is $\delta_{i}$-discretely geodesic, and let $d=\max \left\{1, \delta_{1}, \ldots, \delta_{n}\right\}$. We define a distance on $G$ by stating that $\operatorname{dist}(x, y)$ is the infimum over all $\sum_{i=1}^{n-1} \operatorname{dist}\left(x_{i}, x_{i+1}\right)$, where $x_{1}=x, x_{n}=y$ and for every $i=1,2, \ldots, n-1$ either $x_{i}, x_{i+1}$ are both in the same left coset $g H_{j}$ and $\operatorname{dist}\left(x_{i}, x_{i+1}\right)=\operatorname{dist}_{j}\left(x_{i}, x_{i+1}\right)$, or $x_{i}, x_{i+1}$ are in two different left cosets, coincide with one of the pairs of points minimizing the distance chosen before, and $\operatorname{dist}\left(x_{i}, x_{i+1}\right)$ is the distance $\operatorname{dist}_{S}\left(x_{i}, x_{i+1}\right)$ in the word metric on $G$ corresponding to the set of generators $S$.

Clearly the distance dist is $d$-discretely geodesic. The fact that it is quasi-isometric to dist $_{S}$ follows from the behavior of discrete geodesics of $\left(G\right.$, dist $\left.{ }_{S}\right)$ as described in [DS05b, Lemma 4.28], as well as from the fact that $\operatorname{dist}_{i}, \operatorname{dist}_{S_{i}}$ and the restriction of $\operatorname{dist}_{S}$ to $H_{i}$ are quasi-isometric.

On order to apply Proposition 7.11, it remains to prove that for $x, y \in g H_{i}$ such that $\operatorname{dist}_{i}(x, y)$ is large enough, $\operatorname{dist}(x, y)=\operatorname{dist}_{i}(x, y)$. Indeed, assume that there exists a sequence $x_{1}=x, x_{2}, \ldots, x_{n}=y$ such that either $x_{i}, x_{i+1}$ are both in the same left coset $g H_{j}$, or $x_{i}, x_{i+1}$ are in two different left cosets, coincide with one of the pairs of points minimizing the distance chosen before, and $\sum_{i=1}^{n-1} \operatorname{dist}\left(x_{i}, x_{i+1}\right)<\operatorname{dist}_{i}(x, y)$.

If $x_{i}, x_{i+1}$ are both in the same left coset $g H_{j}$, let $\left[x_{i}, x_{i+1}\right]$ be a discrete geodesic sequence in $\left(g H_{j}\right.$, dist $\left._{j}\right)$. If $x_{i}, x_{i+1}$ are in two different left cosets, and are minimizing the distance, let $\left[x_{i}, x_{i+1}\right]$ be a discrete geodesic sequence in $\left(G, \operatorname{dist}_{S}\right)$.

The assumption that $\sum_{i=1}^{n-1} \operatorname{dist}\left(x_{i}, x_{i+1}\right)<\operatorname{dist}_{i}(x, y)$ implies that $\left[x_{1}, x_{2}\right] \cup \cdots\left[x_{n-1}, x_{n}\right]$ is a quasi-geodesic in $\left(G\right.$, dist $\left._{S}\right)$. We apply Corollary 8.14 in [DS05b] to the quasigeodesics $\left[x_{1}, x_{2}\right]$ and $\left[x_{2}, x_{3}\right] \cup \cdots\left[x_{n-1}, x_{n}\right]$. The hypotheses in the Corollary are satisfies because either $\left[x_{1}, x_{2}\right]$ and $\left[x_{n-1}, x_{n}\right]$ are geodesic sequences minimizing distances between left cosets, or they are geodesic sequences in left cosets, in which case can be applied property $\left.\left(\alpha_{1}\right)\right)$ satisfied by left cosets of peripheral subgroups in relatively hyperbolic groups. It follows that $\operatorname{dist}_{S}\left(x_{1}, x_{n}\right)=\operatorname{dist}_{S}(x, y)$ is bounded uniformly, hence the same holds in the distance dist $_{i}$.

We end up by some considerations concerning the behavior of median spaces and spaces with measured walls with respect to ultralimits and asymptotic cones. For definitions of these notions we refer the reader to [dDW84], [Dru06] and [Dru] for instance.

Proposition 7.14. Any ultralimit of a sequence of median spaces is a median space.

Proof. Let $\left(X_{n}, \operatorname{dist}_{n}\right)$ be a sequence of median spaces, and let $X_{\omega}=\lim ^{\omega}\left(X_{n} ; e_{n}, \operatorname{dist}_{n}\right)$ be an ultralimit of it endowed with the induced metric dist $\omega_{\omega}$. Consider three arbitrary points $a=\lim ^{\omega}\left(a_{n}\right), b=\lim ^{\omega}\left(b_{n}\right)$ and $c=\lim ^{\omega}\left(c_{n}\right)$. If $m_{n}$ is a median point of $a_{n}, b_{n}, c_{n}$ then $m=\lim ^{\omega}\left(m_{n}\right)$ is obviously a median point for $a, b, c$. 
Now let $p$ be any point in $M(a, b, c)$ in $X_{\omega}$. Then $p=\lim ^{\omega}\left(p_{n}\right)$ and $p_{n}$ is in $M_{o(1)}\left(x_{n}, y_{n}, z_{n}\right)$ for $\omega$-almost any $n$. From this and Lemma 2.20 it follows that $\operatorname{dist}_{\omega}(p, m)=$ $\lim _{\omega} \operatorname{dist}\left(p_{n}, m_{n}\right)=0$.

Corollary 7.15. Any asymptotic cone of a median space is a median space.

Remark 7.16. The statement in Corollary 7.15 can be easily improved to the following statement: any asymptotic cone of a $\phi$-strongly quasi-median space, with $\phi(x) \ll x$, is a median space.

Proposition 7.14, Lemma 3.13, Theorem 5.1 and Lemma 3.9 imply that, given a sequence of spaces with measured walls $\left(X_{n}, \mathcal{W}_{n}, \mathcal{B}_{n}, \mu_{n}\right)$ endowed with the induced pseudometrics pdist $_{\mu_{n}}$, any ultralimit $X_{\omega}=\lim ^{\omega}\left(X_{n} ; e_{n}\right.$, pdist $\left._{\mu_{n}}\right)$ of the pseudo-metric spaces $\left(X_{n}\right.$, pdist $\left._{\mu_{n}}\right)$ is a space with measured walls such that the limit metric coincides with the metric coming from the measured wall structure. Nevertheless, there is a priori no relation between the walls thus obtained on $X_{\omega}$ and the walls on each $X_{n}$.

\section{REFERENCES}

[AW81] C.A. Akemann and M.E. Walter, Unbounded negative definite functions, Canad. J. Math. 33 (1981), 862-871.

[Bas01] S. A. Basarab, The dual of the category of generalized trees, Ann. Ştiinţ. Univ. Ovidius Constanţa Ser. Mat. 9 (2001), 1-20.

[Bau01] H. Bauer, Measure and Integration Theory, Studies in Math., vol. 26, de Gruyter, Berlin, 2001.

[BCK66] J. Bretagnolle, D. Dacunha Castelle, and J.-L. Krivine, Lois stables et espaces $L^{p}$, Ann. IHP, section B 2 (1966), no. 3, 231-259.

[BFGM07] U. Bader, A. Furman, T. Gelander, and N. Monod, Property (T) and rigidity for actions on Banach spaces, Acta Math. 198 (2007), 57-105.

[BH83] H.-J. Bandelt and J. Hedlikova, Median algebras, Discrete Math. 45 (1983), 1-30.

[BP03] M. Bourdon and H. Pajot, Cohomologie $l_{p}$ et espaces de Besov, J. Reine Angew. Math. 558 (2003), 85-108.

$\left[\mathrm{CCJ}^{+}\right.$01] P.A. Cherix, M. Cowling, P. Jolissaint, P. Julg, and A. Valette, Groups with the Haagerup Property (Gromov's a-T-menability), Progress in Math., vol. 197, Birkhauser, Boston, 2001.

[Che00] V. Chepoi, Graphs of Some CAT(0) Complexes, Adv. in Appl. Math. 24 (2000), 125-179.

[CMV04] P.A. Cherix, F. Martin, and A. Valette, Spaces with measured walls, the Haagerup property and property (T), Ergod. Th. \& Dynam. Sys. 24 (2004), 1895-1908.

[CN04] I. Chatterji and G. Niblo, From wall spaces to CAT(0) cube complexes, preprint ARXIV:MATH.GT/0309036, 2004.

[dCTV06] Y. de Cornulier, R. Tessera, and A. Valette, Isometric group actions on Banach spaces and representations vanishing at infinity, preprint ARXIV:MATH.RT/0612398, 2006.

[dDW84] L. Van den Dries and A. Wilkie, On Gromov's theorem concerning groups of polynomial growth and elementary logic, J. Algebra 89 (1984), 349-374.

[Del77] P. Delorme, 1-cohomologie des représentations unitaires des groupes de Lie semi-simples et résolubles. Produits tensoriels et représentations, Boll. Soc. Math. France 105 (1977), 281336.

[dlHV89] P. de la Harpe and A. Valette, La propriété (T) de Kazhdan pour les groupes localement compacts, Astérisque, vol. 175, Société Mathématique de France, 1989. 
[Dru] C. Druţu, Quasi-isometry rigidity of groups, preprint, ARXIV:MATH.GT/0612256.

[Dru06]_ Relatively hyperbolic groups: geometry and quasi-isometric invariance, preprint, ARXIV:MATH.GR/0605211, 2006.

[DS05a] C. Druţu and M. Sapir, Relatively hyperbolic groups with rapid decay property, Int. Math. Res. Notices 19 (2005), 1181-1194.

[DS05b] _ Tree-graded spaces and asymptotic cones of groups, Topology 44 (2005), 959-1058, with an appendix by D. Osin and M. Sapir.

[dV84] M. Van de Vel, Dimension of binary convex structures, Proc. London Math. Soc. 48 (1984), no. $3,24-54$.

[dV93] Theory of Convex Structures, Elsevier Science Publishers, Amsterdam, 1993.

[FH74] J. Faraut and K. Harzallah, Distances hilbertiennes invariantes sur un espace homogène, Ann. Institut Fourier 3 (1974), no. 24, 171-217.

[Ger97] V. N. Gerasimov, Semi-splittings of groups and actions on cubings, Algebra, geometry, analysis and mathematical physics (Russian) (Novosibirsk, 1996), Izdat. Ross. Akad. Nauk Sib. Otd. Inst. Mat., Novosibirsk, 1997, pp. 91-109, 190.

[Ger98] V.N. Gerasimov, Fixed-point-free actions on cubings, Siberian Adv. Math. 8 (1998), no. 3, $36-58$.

[Gui77] A. Guichardet, Étude de la 1-cohomologie et de la topologie du dual pour les groupes de Lie à radical abélien, Math. Ann. 228 (1977), no. 1, 215-232.

[Hou74] C. H. Houghton, Ends of locally compact groups and their coset spaces, J. Austral. Math. Soc. 17 (1974), 274-284, Collection of articles dedicated to the memory of Hanna Neumann, VII.

[HP98] F. Haglund and F. Paulin, Simplicité de groupes d'automorphismes d'espaces à courbure négative, The Epstein Birthday Schrift (C. Rourke, I. Rivin, and C. Series, eds.), Geometry and Topology Monographs, vol. 1, International Press, 1998, pp. 181-248.

[HW05] Ch. Hruska and D. Wise, preprint in preparation, 2005.

[Isb80] J. R. Isbell, Median Algebra, Trans. Amer. Math. Soc. (1980), no. 260, 319-362.

[JR06] William B. Johnson and N. Lovasoa Randrianarivony, $l_{p}(p>2)$ does not coarsely embed into a Hilbert space, Proc. Amer. Math. Soc. 134 (2006), no. 4, 1045-1050.

[KPR84] N.J. Kalton, N.T. Peck, and J.W. Roberts, An F-space sampler, London Math. Soc. Lecture Note Series, vol. 89, Cambridge University Press, Cambridge, 1984, xii+240 pp.

[Laf] V. Lafforgue, Un renforcement de la propriété (T), preprint, 2006.

[Nic] B. Nica, Group actions on median spaces, Master Thesis.

[Nic04] _ Cubulating spaces with walls, Alg. \& Geom. Topology 4 (2004), 297-309.

[Nie78] J. Nieminen, The ideal structure of simple ternary algebras, Colloq. Math. 40 (1978), 23-29.

[Now06] P.W. Nowak, Group actions on Banach spaces and a geometric characterization of a-Tmenability, Topology and its Applications 153 (2006), 3409-3412.

[NR97] G. A. Niblo and L. D. Reeves, Groups acting on CAT(0) cube complexes, Geometry and Topology 1 (1997), 7 pp.

[NR98] _ Groups acting on cubes and Kazhdan's property (T), Proc. Amer. Math. Soc. 126 (1998), no. 3, 693-699.

[NSSS05] G. A. Niblo, M. Sageev, P. Scott, and G.A. Swarup, Minimal cubings, Int. J. Algebra Comput. 15 (2005), no. 2, 343-366.

[Pan95] P. Pansu, Cohomologie $L^{p}$ : invariance sous quasi-isométrie, preprint, http://www.math.u-psud.fr/\%7Epansu/liste-prepub.html, 1995.

[Rol98] M. Roller, Poc Sets, Median Algebras and Group Actions, preprint, University of Southampton, http://www.maths.soton.ac.uk/pure/preprints.phtml, 1998.

[RS98] G. Robertson and T. Steger, Negative definite kernels and a dynamical characterization of property (T) for countable groups, Ergod. Th. \& Dynam. Sys. 618 (1998), 247-253. 
[Sag95] M. Sageev, Ends of group pairs and non-positively curved cube complexes, Proc. London Maths. Soc. 71 (1995), no. 3, 585-617.

[Sch38] I.J. Schoenberg, Metric spaces and positive definite functions, Trans. Amer. Math. Soc. 44 (1938), 522-536.

[Sch85] R. Scharlau, A characterization of Tits buildings by metrical properties, J. London Math. Soc. 32 (1985), no. 2, 317-327.

[Sho54a] M. Sholander, Medians and betweenness, Proc. Amer. Math. Soc. 5 (1954), 801-807.

[Sho54b] _ Medians, lattices and trees, Proc. Amer. Math. Soc. 5 (1954), 808-812.

[WW75] J.H. Wells and L.R. Williams, Embeddings and extensions in analysis, Springer-Verlag, New York-Heidelberg, 1975.

[Yu05] G. Yu, Hyperbolic groups admit proper isometric actions on $\ell^{p}$-spaces, Geom. Funct. Anal. 15 (2005), no. 5, 1144-1151.

The Ohio-State University, 231 W18th Ave, 43210 Columbus OH, USA.

E-mail address: indira@math.ohio-state.edu

UFR de Mathématiques, Université de Lille 1, F-59655 Villeneuve d'Ascq Cedex, France, E-mail address: Cornelia.Drutu@math.univ-lille1.fr

Laboratoire de Mathématiques Université de Paris Xi (Paris-Sud), 91405 Orsay, France, E-mail address: frederic.haglund@math.u-psud.fr 